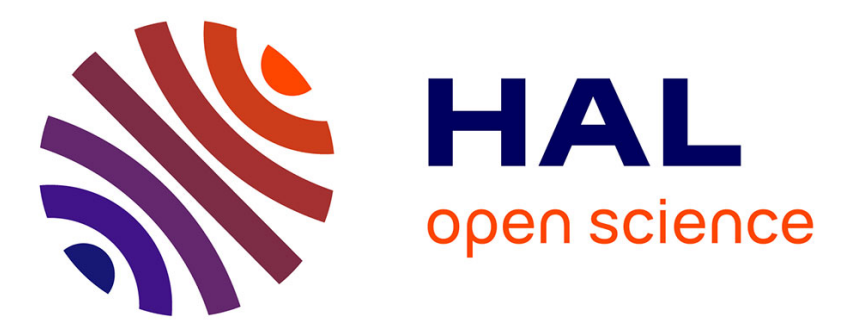

\title{
Artificial intelligence tools and inverse methods for estimating the thermal diffusivity of building materials
}

Stéphane Grieu, Olivier Faugeroux, Adama Traoré, Bernard Claudet, Jean-Luc Bodnar

\section{- To cite this version:}

Stéphane Grieu, Olivier Faugeroux, Adama Traoré, Bernard Claudet, Jean-Luc Bodnar. Artificial intelligence tools and inverse methods for estimating the thermal diffusivity of building materials. Energy and Buildings, 2011, 43 (2-3), pp.543-554. 10.1016/j.enbuild.2010.10.020 . hal-01273275

\section{HAL Id: hal-01273275 \\ https://hal-univ-perp.archives-ouvertes.fr/hal-01273275}

Submitted on 12 Feb 2016

HAL is a multi-disciplinary open access archive for the deposit and dissemination of scientific research documents, whether they are published or not. The documents may come from teaching and research institutions in France or abroad, or from public or private research centers.
L'archive ouverte pluridisciplinaire HAL, est destinée au dépôt et à la diffusion de documents scientifiques de niveau recherche, publiés ou non, émanant des établissements d'enseignement et de recherche français ou étrangers, des laboratoires publics ou privés.

\section{(1) (1) $\$$}

Distributed under a Creative Commons Attribution - NonCommercial - NoDerivatives| 4.0 


\title{
Artificial intelligence tools and inverse methods for estimating the thermal diffusivity of building materials
}

\author{
Stéphane Grieu ${ }^{1, *}$, Olivier Faugeroux ${ }^{1}$, Adama Traoré1, Bernard Claudet ${ }^{1}$ and Jean-Luc Bodnar ${ }^{2}$ \\ ${ }^{1}$ ELIAUS Lab., University of Perpignan, 52 Av. Paul Alduy, 66860, Perpignan, France \\ ${ }^{2}$ GRESPI Lab., University of Reims Champagne-Ardenne, 9 Bd de la Paix, 51100, Reims, France \\ \{grieu; faugeroux; traore; claudet\}@univ-perp.fr; jl.bodnar@univ-reims.fr
}

\begin{abstract}
European energy context highlights the building sector as one of the largest sectors of energy consumption. Consequently, the "Energy Performance of Buildings Directive", adopted in 2002 and focusing on energy use in buildings, requires all the EU members to enhance their building regulations and to introduce energy certification schemes, with the aim of both reducing energy consumption and improving energy efficiency. That is why carrying out an energy performance diagnosis is mandatory, notably when buying or selling properties. Indeed, invisible defaults, like, for example, non-emerging cracks or delaminations, could have a detrimental effect on insulating qualities. Esimaing in-situ thermo-physical properties allowing locating these defaults, the present work focuses on proposing new and efficient approaches based on the use of both artificial intelligence tools (artificial neural networks and neuro-fuzzy systems) and inverse methods for characterizing building materials i.e. for estimating their thermal diffusivity using thermograms obtained thanks to a non-destructive photothermal method.
\end{abstract}

Keywords: energy performance diagnosis, characterization of building materials, thermal diffusivity, non-destructive photothermal methods, inverse methods, artificial neural networks, neuro-fuzzy systems.

\section{Introduction}

The actual European energy context reveals that the building, industry and transport sectors are the three largest sectors of energy consumption. In France, about 25\% of GreenHouse Gases (GHG) emissions and 45\% of energy consumption are due to buildings [1]. Consequently, the adopted "Energy Performance of Buildings Directive" [2], focusing on energy use in buildings, requires all the European Union (EU) members to enhance their building regulations and to introduce energy certification schemes, with the aim of both reducing energy consumption and improving energy efficiency [3,4]. The European Council's ambitious objectives of saving $20 \%$ of the energy consumption compared to projections for 2020 and of reducing of at least $20 \%$ the GHG emissions compared to the 1990 level play a central role in the EU energy policy [5]. Thus, an advanced energy performance diagnosis has to be done [6], notably when buying or selling buildings [7]. Presence of invisible defaults, like non emerging cracks, delaminations or moisture [8], in a wall or a ceiling, completely spoils the insulating qualities of a building [9]. A future owner would be pleased to locate these defaults. In the same way, during the renovation of a building, if a precise draught does not exit, it would be useful to know where are both gas or water ducts and electricity cables, if an old opening has been blocked up, or if a wall or a ceiling is crossed by a wooden beam. Unfortunately, all of these particularities are usually hidden. Whatever the situation, the challenge is the same: being able to locate invisible things under a layer of plaster or similar material, which amounts to locate inhomogeneities in a homogeneous medium. These defaults locally modify the global thermophysical properties of the medium. Thus, an in-situ estimation of these properties can lead us to locate the above-mentioned defaults, making a cartography of the observed medium.

In the present work, properties estimation methods, alternative to classic ones, are proposed using thermograms obtained with a non-destructive photothermal method. Its principle is as

"Corresponding author. Tel.: +334686622 02; Fax: +33468662287. 
follows: the sample to be characterized is excited by a light source and its thermal response, called thermogram, is recorded. From the obtained thermogram, one can estimate several thermophysical properties such as the thermal diffusivity and effusivity or the thickness of a layer for a multi-layer material. These methods can be classified as follows, depending on the time profile of the excitation: one speaks of pulsed methods when the excitation is an impulse and of modulated methods when the excitation is periodic. Pulsed methods are rich in information but impose a high excitation level, which is a problem for fragile or ductile materials. With modulated methods, the excitation is weaker but they give information at the modulation frequency only. A recent solution is to apply an excitation with a random time profile. Previous studies have shown all the interest of this kind of methods [10,11]. Sample properties are usually estimated thanks to wellknown methods (Parker's technique [12] or inverse methods), after rebuilding its impulse response using correlation analysis techniques (the GRESPI laboratory of the University of Reims ChampagneArdenne uses a signal processing method proposed by Jacques Max [13]). Unfortunately, these techniques are very complex to apply, require a large computational time to reach a given precision and are, in some cases, not very efficient. That is why the main objective of the present work is to test some tools belonging to the field of artificial intelligence (artificial neural networks and neuro-fuzzy systems) $[14,15]$ with the aim of rebuilding the impulse response of a sample or for estimating directly its properties from its response to a random excitation. Artificial neural networks, a useful tool for modeling and controlling non-linear systems [16-22], are known as universal and parsimonious approximators. They present some interesting attributes, mostly their learning and generalization capabilities, to be used for rebuilding impulse responses of building materials (in this case, the thermal diffusivity of the concerned materials is thereafter determined by means of inverse methods; one could speak of "neuro-inverse" approach) or for directly estimating the above-mentioned thermophysical properties. As a more in-depth approach and with the objective of combining expert knowledge about characterization of materials, readability, learning ability and connectionist structure, neuro-fuzzy systems were also tested for estimating the thermal diffusivity of building materials; one could speak of "neuro-fuzzy" approach. First, we will present the fundaments of the photothermal experiment and we will focus on the random method (section 2). Inverse method for parameters estimation will be described too. Then, we will be interested in both the simulated data, provided by the GRESPI laboratory, and the artificial intelligence tools we use, i.e. multi-layer artificial neural networks [23] and adaptative network-based fuzzy inference systems [24] (section 3). Finally, we will present the results about impulse responses rebuilding and thermal diffusivities estimation (section 4). We will end this paper by a conclusion and future works (section 5).

\section{The photothermal experiment}

\subsection{Fundamentals}

The photothermal experiment, a non-destructive control method, consists in submitting the sample to be characterized to a light flux. As a consequence, the absorption of light causes a local elevation of temperature. The IR emission is recorded (Figure 1). With low heating hypothesis, the obtained photothermal signal is proportional to the sample surface temperature and is dependent on the thermophysical properties of the observed sample, its structure, the possible presence of defects or delaminations, etc... The temporal profile of the excitation flux is one of the main photothermal experiment characteristics. If the excitation is a pulse (as close to a Dirac as possible), the experiment is called "pulsed method", better known as "flash method" [25-26]. This method is very efficient: the sample response contains all the frequencies of interest. However, using this technique, a high quantity of energy has to be deposited during a very short time lapse. Thus, analyzing fragile materials with the flash method is not possible. On the other hand, if the excitation is periodic (according to a fixed frequency), a sinusoidal profile for example, one speaks of "modulated method". The sample response is then recorded by the way of a lock-in amplifier. In this case, energetic stresses are smaller but the permanent regime has to be reached to begin measuring. The obtained response contains only one frequency and the experiment has to be repeated to obtain a complete study of the sample [27]. The last born of the photothermal methods, called "random method" [28], resorts to a random excitation: commonly, a Pseudo Random Binary Signal (PRBS) is used. 


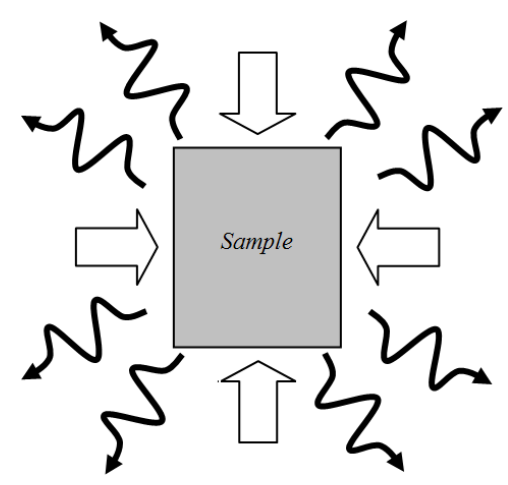

Figure 1. The photothermal experiment. White and black arrows represent the light excitation and the IR response respectively.

\subsection{The random method}

The random method has been developed by the GRESPI laboratory of the University of Reims Champagne-Ardenne and combines elements from both the flash and the modulated methods. Energetic stresses are very low and if the excitation used is perfectly random, the sample response contains all the frequencies of interest. Using correlation analysis techniques, the sample impulse response is recalculated from its response to a random signal. Material properties are identified from the impulse response by well-known techniques [12]. Let us note that the main difficulty in applying the random method is to create experimentally an excitation as close as possible to a real random signal. After that several excitation types have been tested, a PRBS has been chosen to excite samples. Such a signal is composed of low (0) and high (1) states, the duration of which is practically random. Construction of pseudo random sequences consists in getting the output signal of a shift register with a feedback via a modulo-2 addition [29]. Samples can be stressed with a laser diode piloted by a PRBS. IR responses are recorded using an InSb or $\mathrm{HgCdTe}$ infrared detector. In parallel, a model of the response has been developed. Thus, simulating the experiment is possible. Up to now, to obtain the impulse response $\left(R_{\text {imp }}\right)$ from the sample response to a random excitation $\left(R_{\text {rand }}\right)$, the GRESPI laboratory uses the Jacques Max's technique [13]:

$$
R_{i m p}(t)=F T^{-1}\left[\frac{R_{\text {rand }}(f) \cdot E(f)}{E(f) \cdot E(f)}\right][E q .1]
$$

with $F T^{-1}$ the inverse Fourier transform, $R_{\text {rand }}(f)$ the Fourier transform of $R_{\text {rand }}(t)$ and $E(f)$ the Fourier transform of the excitation. The aim of this paper is to show that correlation analysis techniques can be efficiently replaced by artificial intelligence tools such as multi-layer artificial neural networks or neuro-fuzzy systems.

\section{Materials and methods}

\subsection{Database}

The used database is composed of responses to PRBS, impulse responses and thermophysical properties for the following seven building materials: glass wool, concrete, brick, glass, plaster, granite and stainless steel. Responses to a PRBS (Figure 2) and impulse responses (Figure 3) are both composed of 255 points (uniformly spaced in time, $\Delta t=3 \mathrm{~s}$ for responses to PRBS while $\Delta t=$ $3 \times 10^{-2} \mathrm{~s}$ for impulse responses). Table 1 highlights some of the main properties of the seven considered materials: density $(\rho)$, ranging between $200 \mathrm{~kg} / \mathrm{m}^{3}$ and $7900 \mathrm{~kg} / \mathrm{m}^{3}$, calorific capacity $\left(C_{p}\right)$, ranging between $670 \mathrm{~J} / \mathrm{kg} . \mathrm{K}$ and $960 \mathrm{~J} / \mathrm{kg} . \mathrm{K}$, thermal conductivity $(k)$, ranging between 0.04 $\mathrm{W} / \mathrm{m} . \mathrm{K}$ and $16 \mathrm{~W} / \mathrm{m} . \mathrm{K}$, thermal diffusivity $(a)$, ranging between $3 \times 10^{-7} \mathrm{~m}^{2} / \mathrm{s}$ and $4 \times 10^{-5} \mathrm{~m}^{2} / \mathrm{s}$, and thermal effusivity $(b)$, ranging between $73.21 \mathrm{~J} / \mathrm{m}^{2} . \mathrm{K} \mathrm{s}^{1 / 2}$ and $8028.95 \mathrm{~J} / \mathrm{m}^{2} . \mathrm{K} \cdot \mathrm{s}^{1 / 2}$. With the aim of developing the most effective tools, a preliminary study about thermal behaviour has been carried out and allowed choosing the materials used to train or to validate both artificial neural networks and neuro-fuzzy systems. As a conclusion of this study, glass wool, concrete, glass and stainless 
steel were considered as training materials while brick, plaster and granite were used for validating the developed models. Let us note that, among all these materials, only glass wool is an organic material and, as such, presents a very specific thermal behaviour.

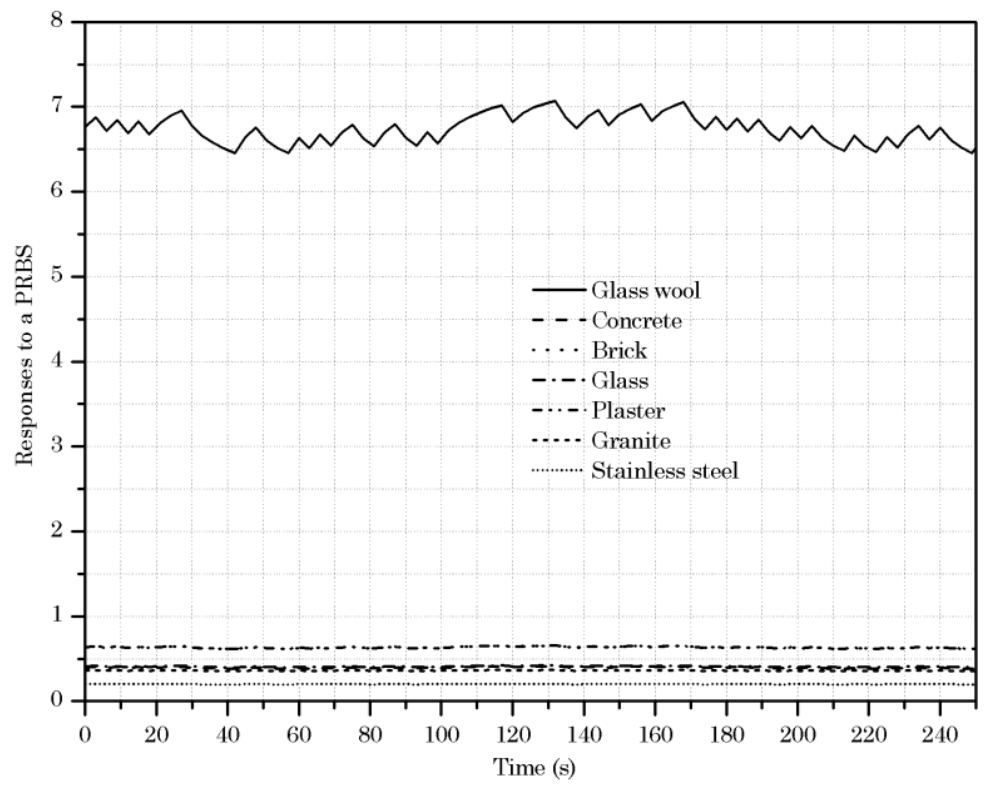

Figure 2. Responses to a PRBS of the seven considered building materials (linear scale).

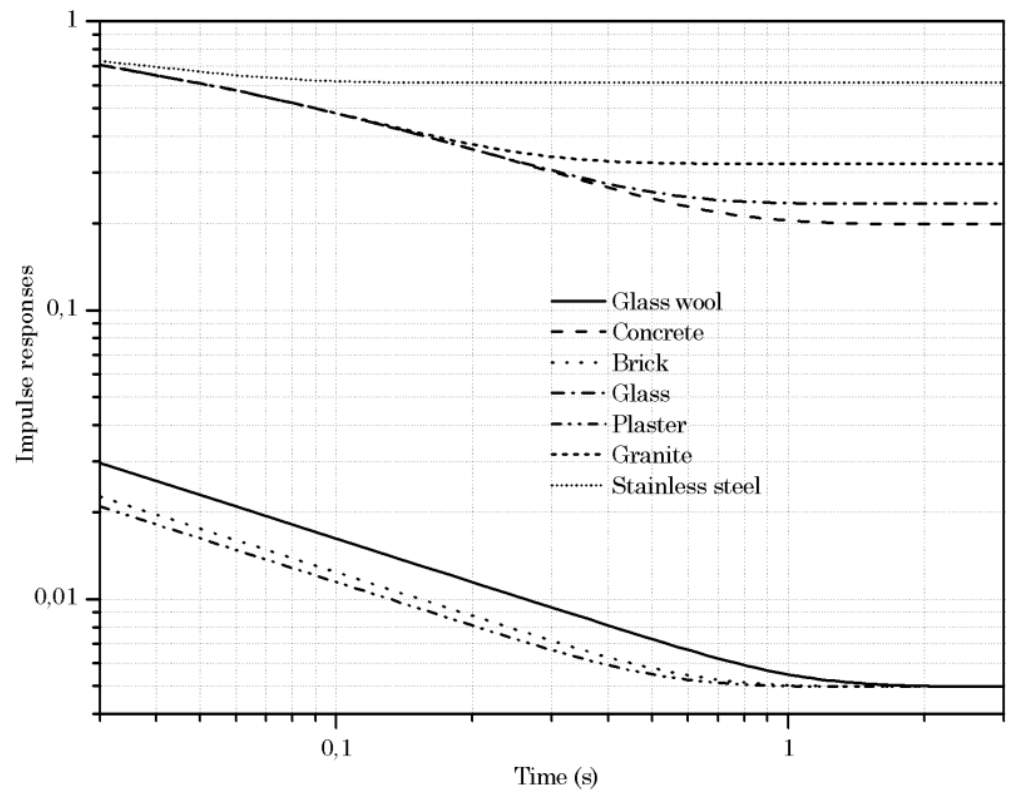

Figure 3. Impulse responses of the seven considered building materials (log-log scale).

Table 1. Materials properties (bibliography values).

\begin{tabular}{cccccc}
\hline Material & $\rho\left[\mathrm{kg} / \mathrm{m}^{3}\right]$ & $C_{p}[\mathrm{~J} / \mathrm{kg} . \mathrm{K}]$ & $k[\mathrm{~W} / \mathrm{m} . \mathrm{K}]$ & $a\left[\mathrm{~m}^{2} / \mathrm{s}\right]$ & $b\left[\mathrm{~J} / \mathrm{m}^{2} .{\left.\mathrm{K} . \mathrm{s}^{1 / 2}\right]}^{-}\right.$ \\
\hline Glass wool & 200 & 670 & 0.04 & $3 \times 10^{-7}$ & 73.21 \\
\hline Concrete & 2300 & 960 & 0.92 & $4.2 \times 10^{-7}$ & 1425.26 \\
\hline Brick & 1600 & 840 & 0.69 & $5.13 \times 10^{-7}$ & 963 \\
\hline Glass & 2530 & 840 & 1.2 & $5.8 \times 10^{-7}$ & 1596.95 \\
\hline Plaster & 1600 & 830 & 0.8 & $6 \times 10^{-7}$ & 1030.73 \\
\hline Granite & 2600 & 870 & 2.5 & $1.1 \times 10^{-6}$ & 2378.02 \\
\hline Stainless steel & 7900 & 510 & 16 & $4 \times 10^{-6}$ & 8028.95 \\
\hline
\end{tabular}




\subsection{Parameter estimation by inverse methods}

\subsubsection{General principle}

The impulse response can be exploited using Parker's technique [12] or by inverse methods [30] to identify thermophysical properties. We usually use inverse methods. The principle of an inverse method is to compare a model to experimental measurements. The model depends on parameters which are usually thermophysical properties combinations. The goal is to minimize a criterion by adjusting the parameters by an iterative process. Calculation is initiated by a priori parameters chosen by the user. Thus, having a mathematical model very close to the used experiment is crucial. First, let us note that bold characters represent vectorial quantities or matrices. Measurements are compared to values obtained by a model function of parameters. So, a comparison criterion (or cost function) can be calculated. The criterion value is minimized by adjusting step by step the parameters values and by computing the new values of the model. The process is stopped when the criterion reaches a critical value, parameters does not change anymore. Thus, we get the estimated values $(\boldsymbol{b})$ of the searched parameters $(\boldsymbol{\beta})$ (Figure 4).

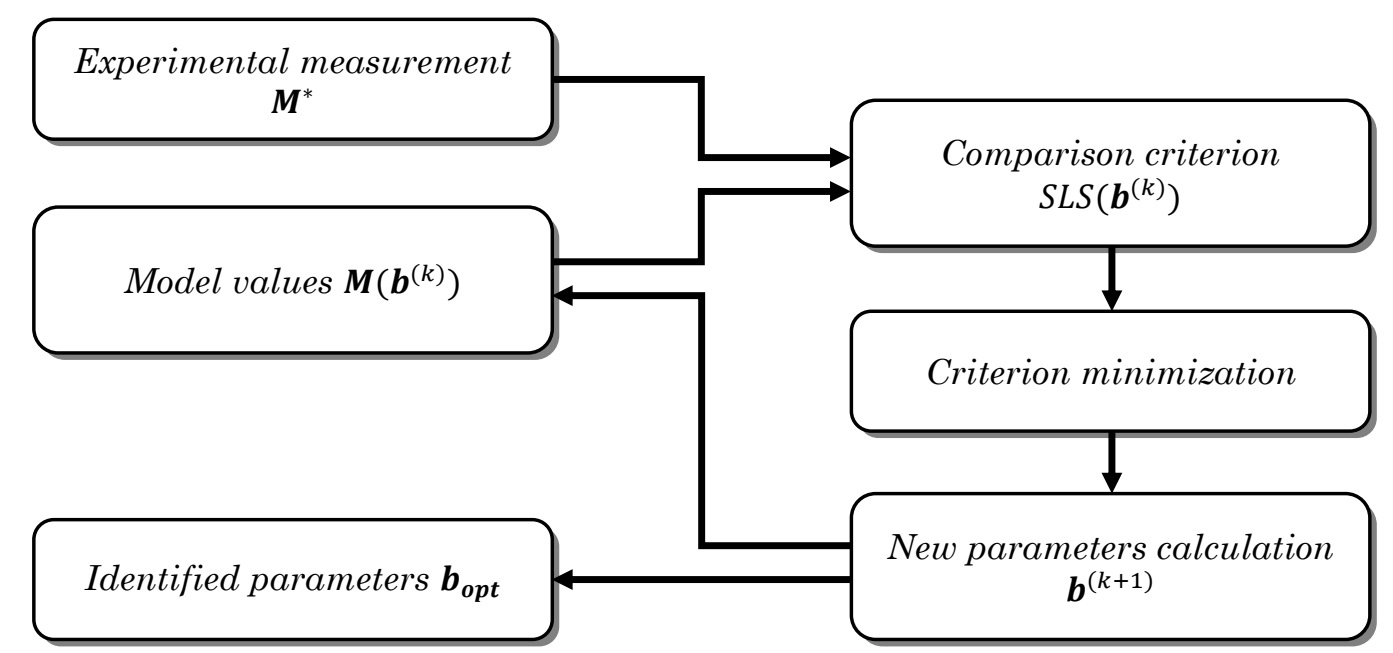

Figure 4. Parameter estimation by inverse methods.

\subsubsection{Comparison criterion}

First, let $\boldsymbol{M}^{*}=\left(M_{1}^{*}, \ldots, M_{k}^{*}, \ldots, M_{n}^{*}\right)^{T}$ be a vector composed of $n$ experimental measurements uniformly spaced in time between $t_{1}$ and $t_{n}$. Next, let $\boldsymbol{M}(\boldsymbol{\beta})=\left(M_{1}, \ldots, M_{k}, \ldots, M_{n}\right)^{T}$ be the model values vector with $M_{k}=\eta\left(t_{k}, \boldsymbol{\beta}\right), \boldsymbol{\beta}$ being the parameters to be identified. Finally, let $S L S(\boldsymbol{\beta})$ be an objective function, defined as the sum of the least square of $\boldsymbol{M}^{*}$ and $\boldsymbol{M}(\boldsymbol{\beta})$, to be minimized with respect to the unknown $\boldsymbol{\beta}$. It could be written [31]:

$$
S L S(\boldsymbol{\beta})=\sum_{k=1}^{n}\left[M_{k}^{*}-M_{k}\right]^{2}=\left[\boldsymbol{M}^{*}-\boldsymbol{M}(\boldsymbol{\beta})\right]^{T} \cdot\left[\boldsymbol{M}^{*}-\boldsymbol{M}(\boldsymbol{\beta})\right][E q .2]
$$

So, $\operatorname{SLS}(\boldsymbol{\beta})$ is a scalar function of the searched parameters $(\boldsymbol{\beta})$. Searching the best estimate $\left(\boldsymbol{b}_{\text {opt }}\right)$ of $\boldsymbol{\beta}$ is searching the $S L S$ function minimum.

\subsubsection{Sensitivity coefficients}

The sensitivity coefficient $X_{p}$ related to a parameter $\beta_{p}$ is given by the first derivative of the model $\eta(t, \boldsymbol{\beta})$ with respect to the just-mentioned parameter. This coefficient depicts the influence of the parameter $\beta_{p}$ on the model:

$$
X_{p}(t, \boldsymbol{\beta})=\left.\frac{\partial \eta(t, \boldsymbol{\beta})}{\partial \beta_{p}}\right|_{\beta_{q \neq p}}[E q \cdot 3]
$$




\subsubsection{Criterion minimization method}

The criterion minimization method depends on the model linearity versus the parameters. We use methods belonging to the gradient methods class: Gauss-Newton's, Box-Kanemasu's and modified Box-Kanemasu's methods [31]. These methods optimize the slope step around the solution. General principle is represented by Figure 4. Model values are firstly calculated with initial parameters, chosen a priori, then, the first criterion value is calculated. If the stopping condition is not satisfied, the algorithm calculates the parameter corrections to make the criterion $S L S(\boldsymbol{\beta})$ decreases at the next iteration. New parameters are used to calculate new model values and to resume the process. A recurrence relationship can be written between respective parameter values at $k^{t h}$ and $(k+1)^{t h}$ iterations, with $\Delta b_{i}^{(k)}$ the corrective step applied to parameter $b_{i}$ of $\boldsymbol{b}$ at $k^{\text {th }}$ iteration:

$$
b_{i}^{(k+1)}=b_{i}^{(k)}+\Delta b_{i}^{(k)}[E q .4]
$$

We frequently use the following criterion (Eq.5), proposed by Beck [31], as stopping condition. If this criterion is satisfied, it means $b_{i}$ has been corrected by a negligible value at $k^{\text {th }}$ iteration:

$$
\frac{\left|b_{i}^{(k+1)}-b_{i}^{(k)}\right|}{\left|b_{i}^{(k)}\right|+\delta_{1}}<\delta \text { with } \delta_{1}=10^{-10} \text { and } \delta=10^{-4} \quad[\text { Eq. } 5]
$$

\subsubsection{Gauss-Newton's method}

The Gauss-Newton's method is one of the simplest and most efficient minimization methods. The Gauss-Newton's step $\Delta \boldsymbol{b}_{G N}^{(k)}$ is given by the following expression:

$$
\Delta \boldsymbol{b}_{G N}^{(k)}=\left[\boldsymbol{X}^{T(k)} \cdot \boldsymbol{X}^{(k)}\right]^{-1} \cdot\left[\boldsymbol{X}^{T(k)} \cdot\left(\boldsymbol{M}^{*}-\boldsymbol{M}\left(\boldsymbol{b}^{(k)}\right)\right)\right][E q \cdot 6]
$$

Let us note that the Hessian approximation $\boldsymbol{X}^{T(k)} \cdot \boldsymbol{X}^{(k)}$ has to be inversed and needs to be wellconditioned. So, $\boldsymbol{X}^{(k)}$ values must be maximum, small values leading to an ill-conditioned matrix and, as a consequence, the inverse algorithm will not converge. Indeed, the efficiency of the GaussNewton's method is dependent on the problem conditioning. Ill-conditioned problems need an adapted method such as the Box-Kanemasu's method.

\subsubsection{Box-Kanemasu's method}

The Gauss-Newton's method is based on a linear approximation of the model. If this hypothesis is really wrong, calculated corrections can oscillate with growing amplitude and the algorithm cannot converge. The Box-Kanemasu's step $\Delta \boldsymbol{b}_{B K}^{(k)}$ is calculated as follows:

$$
\Delta \boldsymbol{b}_{B K}^{(k)}=h_{B K}^{(k)} \cdot \Delta \boldsymbol{b}_{G N}^{(k)}[E q .7]
$$

Taking a look at Eq.7, one can note that a coefficient $h_{B K}$ is assigned to the Gauss-Newton's step $\Delta \boldsymbol{b}_{G N}^{(k)}$. This coefficient is obtained thanks to the procedure depicted by Figure 5. A has a constant value of 1.1 while $g^{(k)}$ is calculated at each iteration in the following way:

$$
g^{(k)}=\left[\Delta \boldsymbol{b}_{G N}^{(k)}\right]^{-1} \cdot\left[\boldsymbol{X}^{T(k)} \cdot \boldsymbol{X}^{(k)}\right] \cdot\left[\Delta \boldsymbol{b}_{G N}^{(k)}\right]^{-1} \quad[E q .8]
$$

\subsubsection{Modified Box-Kanemasu's method: Bard's method}

The Box-Kanemasu's method does not verify if $S L S(\boldsymbol{\beta})$ decreases at the next iteration. That is why Bard modified the method, calculating differently the coefficient $h_{B K m}^{(k)}$ assigned to the GaussNewton's step $\Delta \boldsymbol{b}_{G N}^{(k)}$, as depicted by Figure 6 [31]. So:

$$
\Delta \boldsymbol{b}_{B K m}^{(k)}=h_{B K m}^{(k)} \cdot \Delta \boldsymbol{b}_{G N}^{(k)}[E q .9]
$$




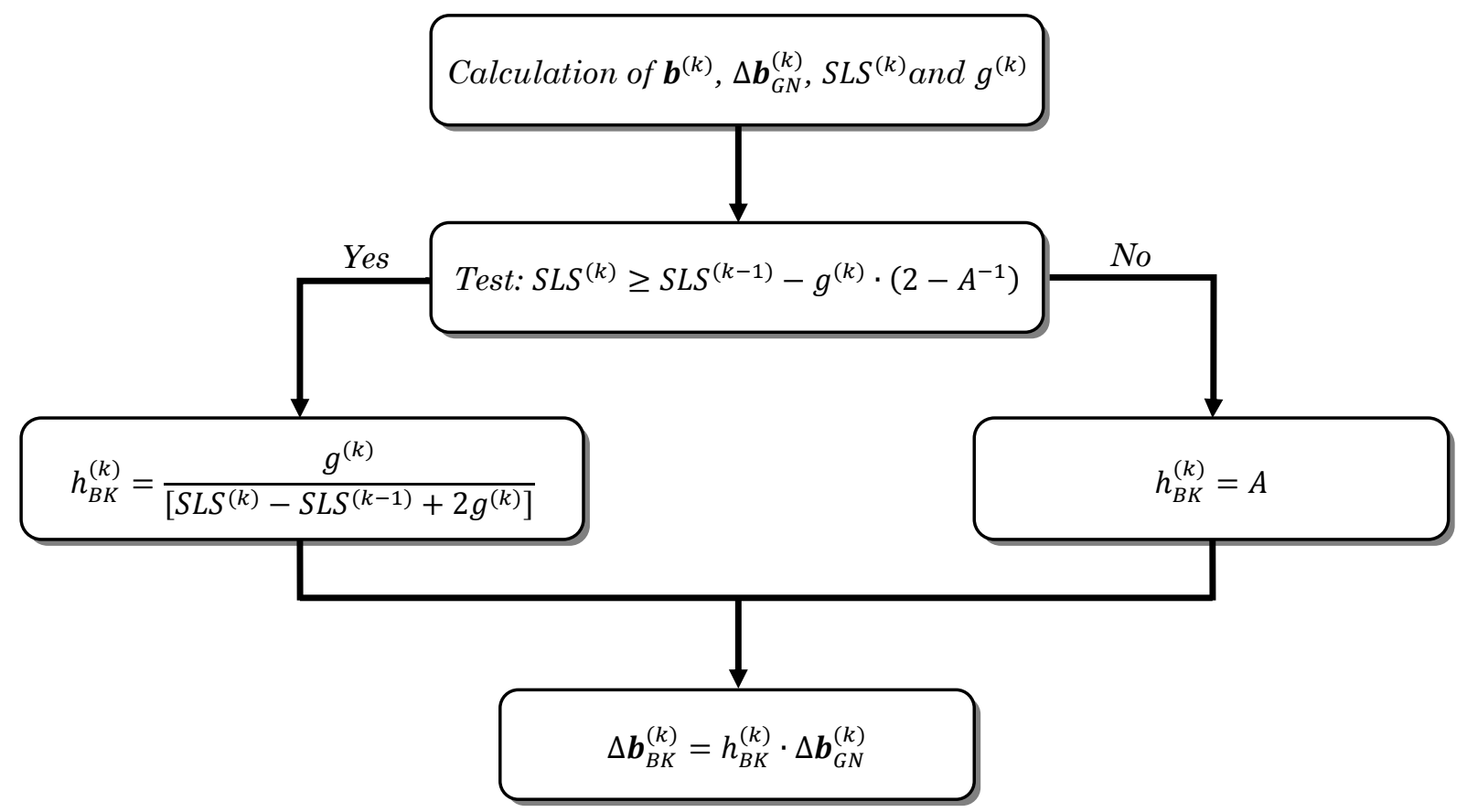

Figure 5. Calculation of $\Delta \boldsymbol{b}_{B K}^{(k)}$.

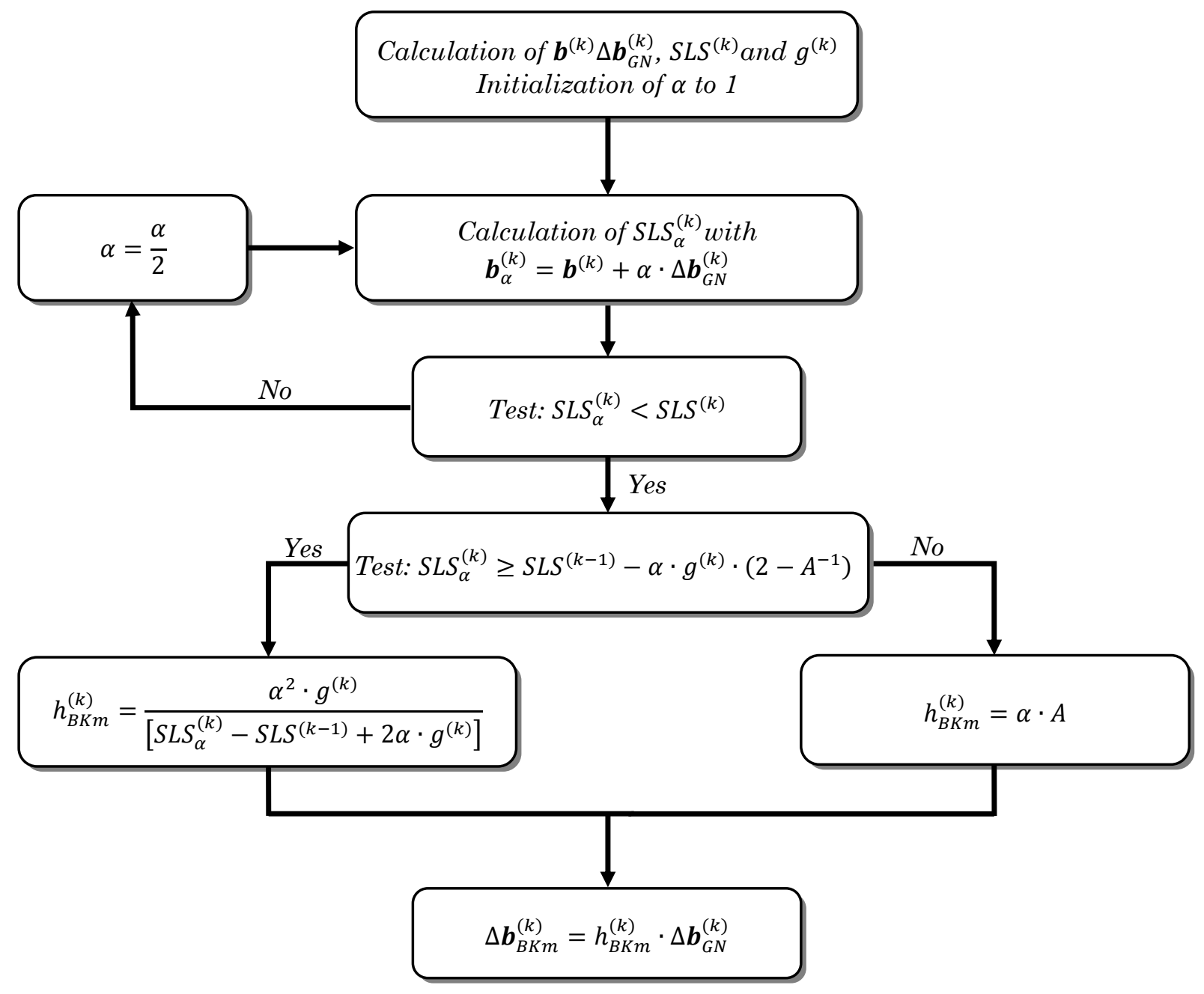

Figure 6. Calculation of $\Delta \boldsymbol{b}_{B K m}^{(k)}$. 


\subsection{Multi-layer artificial neural networks}

Two kinds of multi-layer artificial neural networks have been used for estimating, directly or indirectly, the thermal diffusivity of building materials, with the aim of developing a new and effective approach contributing to the energy performance diagnosis of buildings: the commonlyused Multi-Layer Perceptron (MLP) and the Elman recurrent network. Both networks' topology and the training algorithm used will be described summarily in the present section of the paper.

\subsubsection{The multi-layer Perceptron}

The Perceptron, the simplest neural network, is only able to classify data into two classes [32]. Basically it consists of a single neuron with a number of adjustable weights [33]. It uses an adaptative learning rule. Given a problem which calls for more than two classes, several Perceptrons can be combined: the simplest form of a layered network just has an input layer of source nodes that connect to an output layer of neurons. The single-layer Perceptron can only classify linearly separable problems. For non-separable problems it is necessary to use more layers. A multi-layer network has one or more hidden layers whose neurons are called hidden neurons. The network is fully connected, every node in a layer being connected to all nodes in the next layer. According to the previous remarks, the first network used for the present work is a multi-layer Perceptron, one speaks of feedforward networks. It consists of one layer of linear output neurons and one hidden layer of nonlinear neurons [34] (Figure 7). According to previous tests, more than one hidden layer proved to cause slower convergence during the learning phase because intermediate neurons not directly connected to output neurons learn very slowly. Based on the principle of generalization versus convergence, both number of hidden neurons and iterations completed during the training phase were optimized [19]. The multi-layer Perceptron neural network learns using an algorithm called backpropagation. During this iterative process, input data are repeatedly presented to the network. At each iteration: (1) the entire input sequence is presented to the network, and its outputs are calculated and compared with the target sequence to generate an error sequence; (2) for each time step, the error is backpropagated to find gradients of errors for each weight and bias. This gradient is actually an approximation since the contributions of weights and biases to errors via the delayed recurrent connection are ignored; (3) this gradient is then used to update the weights with a backpropagation training algorithm like the Levenberg-Marquardt algorithm [35-36].

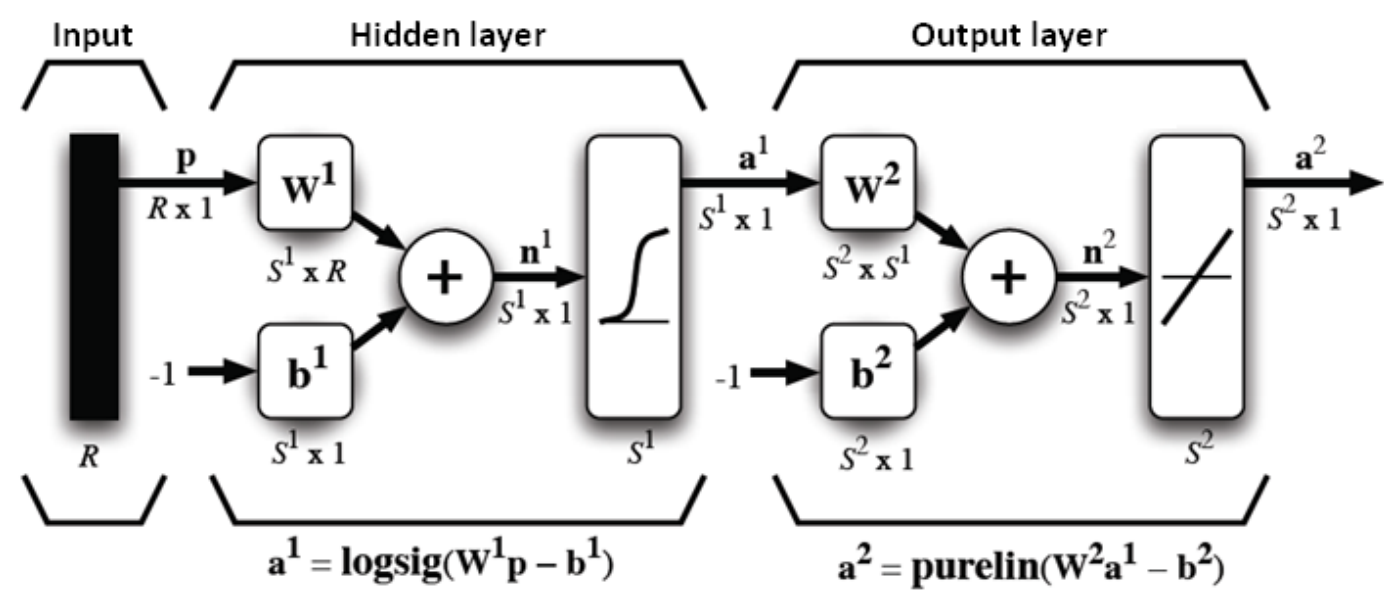

Figure 7. Topology of the multi-layer Perceptron used for estimating the thermal diffusivity of building materials.

\subsubsection{The Elman recurrent network}

Feedforward neural networks have been successfully used to solve problems that require the computation of a static function i.e. a function whose output depends only on the current input, and not on any previous inputs. In the real world however, one encounters many problems which cannot be solved by learning a static function because the function being computed changes with 
each input received. It should be clear from the architecture of feedforward neural networks that past inputs have no way of influencing the processing of future inputs. This situation can be rectified by the introduction of feedback connections in the network [37]. Now network activation produced by past inputs can cycle back and affect the processing of future inputs. The class of neural networks, which contain cycles or feedback connections, is called recurrent neural networks. The Elman network used for rebuilding impulse responses of building materials is a 2layer network with feedback from the first-layer output to the first layer input [38]. This recurrent connection allows this kind of network to both detect and generate time-varying patterns. The Elman network differs only from conventional 2-layer networks, such as the just-described multilayer Perceptron, in that the first layer has a recurrent connection (Figure 8). The delay in this connection stores values from the previous time step, which can be used in the current time step. Because the network can store information for future reference, it is able to learn temporal patterns as well as spatial patterns. The Elman recurrent network can be trained, using an iterative process, to respond to, and to generate, both kinds of patterns. Just as the multi-layer Perceptron, it can approximate any function (with a finite number of discontinuities) with arbitrary accuracy. The only requirement is that its hidden layer must have enough neurons. More hidden neurons are needed as the function being fitted increases in complexity.

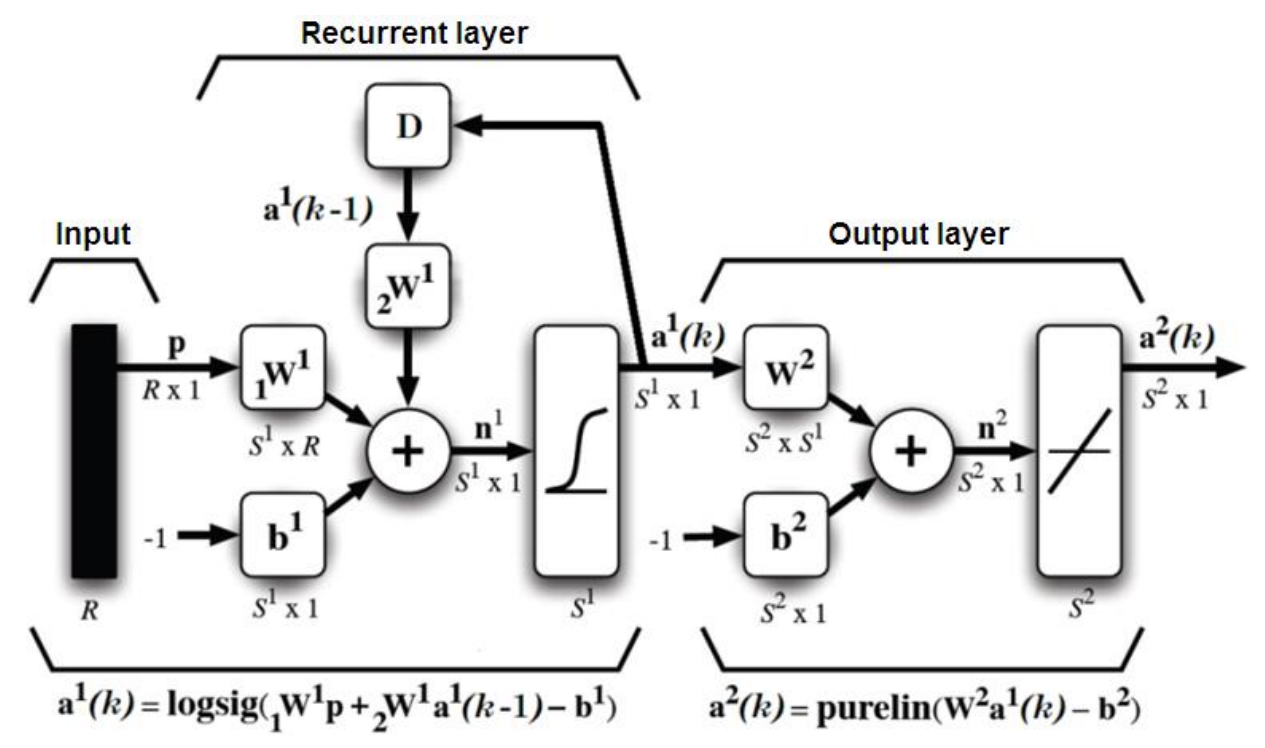

Figure 8. Topology of the Elman recurrent network used for rebuilding impulse responses of building materials.

\subsubsection{The Levenberg-Marquardt algorithm}

Several training methods were used, but the Levenberg-Marquardt algorithm [39] proved to be the fastest and the most robust, whatever the neural network considered. It is particularly adapted for networks of moderate size and has memory reduction feature for use when the training set is large. Like the quasi-Newton methods, the Levenberg-Marquardt algorithm was designed to approach second-order training speed without having to compute the Hessian matrix. When the performance function has the form of a sum of squares, then the Hessian matrix can be approximated as:

$$
H=J^{T} \cdot J[E q .10]
$$

The gradient can be computed as:

$$
g=J^{T} \cdot e[\text { Eq.11] }
$$

where $J$ is the Jacobian matrix that contains first derivatives of the network errors with respect to the weights and biases, and $e$ is a vector of network errors. The Jacobian matrix can be computed through a standard backpropagation technique that is much less complex than computing the 
Hessian matrix. The Levenberg-Marquardt algorithm uses this approximation to the Hessian matrix in the following Newton-like update:

$$
x_{k+1}=x_{k}-\left[J^{T} \cdot J+\mu \cdot I\right]^{-1} \cdot J^{T} \cdot e[E q .12]
$$

When the scalar $\mu$ is zero, this is just Newton's method, using the approximate Hessian matrix. When $\mu$ is large, this becomes gradient descent with a small step size. Newton's method is faster and more accurate near an error minimum, so the aim is to shift towards Newton's method as quickly as possible. Thus, $\mu$ is decreased after each successful step and is increased only when a tentative step would increase the performance function. In this way, the performance function will always be reduced at each iteration of the algorithm. The main drawback of the LevenbergMarquardt algorithm is that it requires the storage of some matrices that can be quite large for certain problems. The size of the Jacobian matrix is $Q \times n$, where $Q$ is the number of training sets and $n$ is the number of weights and biases in the network. It turns out that this matrix does not have to be computed and stored as a whole. For example, if we were to divide the Jacobian into two equal submatrices we could compute the approximate Hessian matrix as follows:

$$
H=J^{T} \cdot J=\left[\begin{array}{ll}
J_{1}^{T} & J_{2}^{T}
\end{array}\right] \cdot\left[\begin{array}{c}
J_{1} \\
J_{2}
\end{array}\right]=J_{1}^{T} \cdot J_{1}+J_{2}^{T} \cdot J_{2}[E q .13]
$$

Therefore, the full Jacobian does not have to exist at one time. The approximate Hessian can be computed by summing a series of subterms. Once one subterm has been computed, the corresponding submatrix of the Jacobian can be cleared.

\subsection{Neuro-Fuzzy Systems (NFS)}

In the field of artificial intelligence, neural networks and fuzzy logic can be combined in neurofuzzy systems in order to achieve both properties of readability and learning ability. Neuro-fuzzy systems synergizes the two techniques by combining the human-like reasoning style of fuzzy systems (through the use of fuzzy sets and a linguistic model consisting of a set of if-then fuzzy rules) with the learning and connectionist structure of neural networks [40,41]. Various neurofuzzy architectures can be found in the scientific literature [42,43], one can mention: NEFCLASS (classification), FALCON and GARIC (process control), ANFIS (process control, signal processing, non-linear approximation, identification)...etc. Because ANFIS is a widely-used and powerful architecture, it has been chosen for estimating the thermal diffusivity of building materials, taking into account expert knowledge.

\subsubsection{Fuzzy if-then rules}

Fuzzy if-then rules are expressions of the form if $A$ then $B$, where $A$ and $B$ are labels or fuzzy sets characterized by appropriate membership functions. Due to their concise form and through the use of linguistic labels and membership functions, fuzzy if-then rules are often employed to capture the imprecise and subjective modes of reasoning that play a central role in the human ability to make decisions in an uncertain environment $[44,45]$. Another form of fuzzy if-then rule, proposed by Takagi and Sugeno, has fuzzy sets involved only in the premise part, the consequent part being described by a non-fuzzy equation of the input variable [46]. Both types of fuzzy if-then rules have been used extensively for modelling and controlling systems. Due to the qualifiers on the premise parts, each fuzzy if-then rule of a set of rules can be considered as a local description of the studied system under consideration.

\subsubsection{Fuzzy Inference Systems (FIS)}

Fuzzy inference systems are also known as fuzzy-rule-based systems or fuzzy controllers when used as controllers. Basically, a fuzzy inference system is composed of five functional blocks [24]: (1) a collection of fuzzy if-then rules; (2) a database which defines the membership functions of the fuzzy sets used to design the fuzzy rules; (3) a decision-making unit allowing performing the inference operations on the rules; (4) a fuzzification interface which transforms the crisp inputs into degrees of match with linguistic values; (5) a defuzzification interface which transforms the 
fuzzy results of the inference into a crisp output. The steps of fuzzy reasoning performed by fuzzy inference systems can be described as follows: (1) the fuzzification step during which the input variables are compared with the membership functions on the premise part to obtain the membership values of each linguistic label; (2) the combination of the membership values on the premise part to get the weight of each rule of the rule base; (3) the generation of the qualified consequent (fuzzy or crisp) of each rule depending on the weight; (4) the defuzzification step during which the qualified consequents are aggregated to produce a crisp output $[47,48]$.

\subsubsection{Adaptative Network-based Fuzzy Inference Systems (ANFIS)}

The acronym ANFIS derives from Adaptative Network-based Fuzzy Inference System. A network-type structure, similar to that of artificial neural networks, which maps inputs through input membership functions and associated parameters and then through output membership functions and associated parameters to output, can be used to interpret an input/output map. The parameters associated with the membership functions changes through the learning process. The adjustment of these parameters is facilitated by a gradient vector. This gradient vector provides a measure of how well the fuzzy inference system is modelling the input/output data for a given set of parameters. When the gradient vector is obtained, any of several optimization routines can be applied in order to adjust the parameters to reduce some error measure [49].

\subsubsection{ANFIS architecture}

For simplicity, we assume, first, that the considered fuzzy inference system has two inputs $x$ and $y$ and one output $z$ and, secondly, that the rule base contains only two fuzzy if-then rules of Takagi and Sugeno's type [46]. The rules are designed in the following way:

First rule: If $x$ is $A_{1}$ AND y is $B_{1}$ THEN $f_{1}=p_{1} x+q_{1} y+r_{1}$

Second rule: If $x$ is $A_{2}$ AND $y$ is $B_{2}$ THEN $f_{2}=p_{2} x+q_{2} y+r_{2}$

Implementing both rules requires the 5-layer ANFIS architecture shown in Figure 9 [24]. A membership function $\mu_{A_{i}}(x)$ or $\mu_{B_{i}}(y)$, specifying the degree of which $x$ satisfies $A_{i}$ or $y$ satisfies $B_{i}$, with $i=1,2$, is associated with every node in the first layer. Usually $\mu_{A_{i}}(x)$ and $\mu_{B_{i}}(y)$ are chosen to be bell-shaped with a minimum and a maximum equal to 0 and 1 respectively:

$$
\begin{aligned}
& \mu_{A_{i}}(x)=\frac{1}{1+\left[\left(\frac{x-c_{i}}{a_{i}}\right)^{2}\right] b_{i}} \text { or } \mu_{A_{i}}(x)=\exp \left[-\left(\frac{x-c_{i}}{a_{i}}\right)^{2}\right][\text { Eq.14] } \\
& \mu_{B_{i}}(y)=\frac{1}{1+\left[\left(\frac{x-c_{i}^{\prime}}{a_{i}^{\prime}}\right)^{2}\right] b_{i}^{\prime}} \text { or } \mu_{B_{i}}(y)=\exp \left[-\left(\frac{x-c_{i}^{\prime}}{a_{i}^{\prime}}\right)^{2}\right][\text { Eq.15] }
\end{aligned}
$$

with $\left\{a_{i}, b_{i}, c_{i}\right\}$ and $\left\{a_{i}^{\prime}, b_{i}^{\prime}, c_{i}^{\prime}\right\}$ two parameter sets. As the values of these parameters change, the bell-shaped functions vary accordingly, thus exhibiting various forms of membership functions on linguistic label $A_{i}$. Any continuous and piecewise differentiable functions, such as commonly-used trapezoidal or triangular-shaped membership functions, can be used. Nodes in the second layer evaluate the premises of the rules, multiplying the incoming signals and sending the product out. So, the $i^{\text {th }}$ node output represents the firing strength of rule $i$. Let us note that many other T-norm operators, allowing performing generalized AND, can be used in this layer [50].

$$
\omega_{i}=\mu_{A_{i}}(x) \times \mu_{B_{i}}(y) \text { with } i=1,2 \quad \text { [Eq.16] }
$$

The $i^{\text {th }}$ node in the third layer calculates the ratio of the $i^{\text {th }}$ rule's firing strength to the sum of all rules' firing strengths (i.e. the contribution of the $i^{\text {th }}$ rule), such as:

$$
\bar{\omega}_{i}=\frac{\omega_{i}}{\omega_{1}+\omega_{2}} \text { with } i=1,2 \text { [Eq. 17] }
$$


Nodes in the fourth layer evaluate the conclusions of the rules. So, the $i^{\text {th }}$ node evaluates the conclusion of the $i^{\text {th }}$ rule, with $\bar{\omega}_{i}$ the output of layer 3 and $\left\{p_{i}, q_{i}, r_{i}\right\}$ a parameter set. Parameters in this layer can be referred as consequent or conclusion parameters. So:

$$
\bar{\omega}_{i} f_{i}=\bar{\omega}_{i}\left(p_{i} x+q_{i} y+r_{i}\right) \text { with } i=1,2 \text { [Eq.18] }
$$

Finally, the single node in the fifth and last layer computes the overall output as the summation of all incoming signals. It is observed that given the values of premise parameters, the overall output can be expressed as a linear combination of the consequent parameters:

$$
f=\sum_{i} \bar{\omega}_{i} f_{i}=\frac{\sum_{i} \omega_{i} f_{i}}{\sum_{i} \omega_{i}} \text { with } i=1,2 \quad \text { [Eq. 19] }
$$

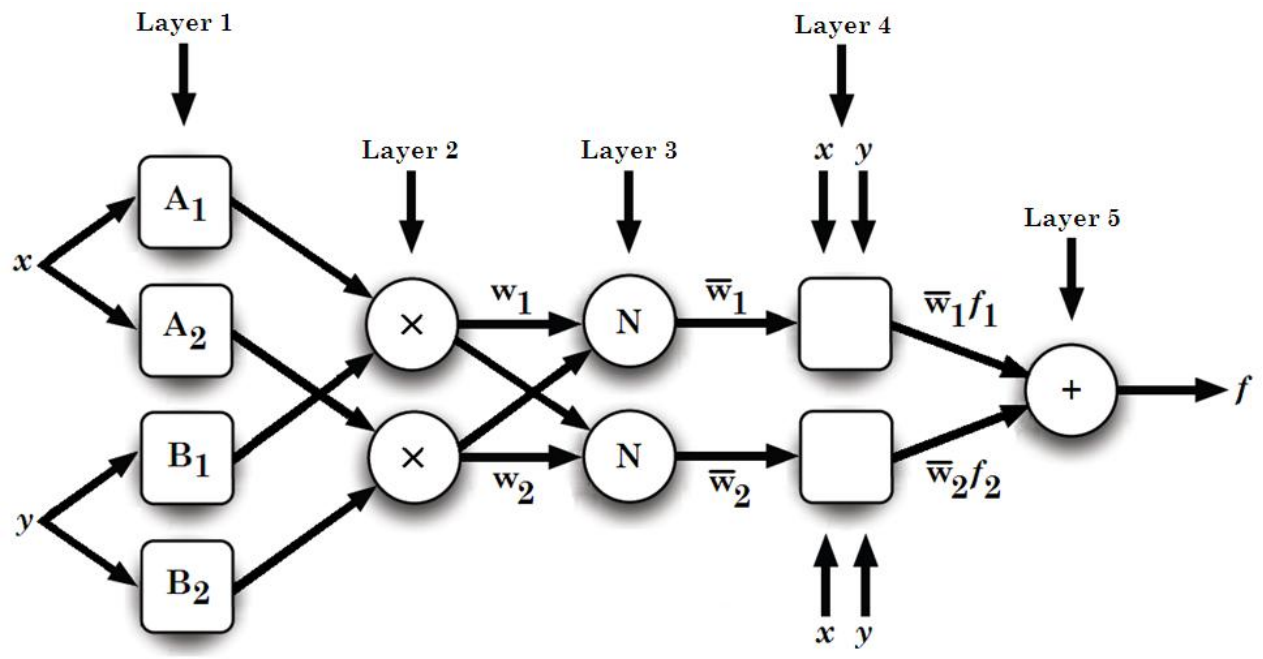

Figure 9. 5-layer adaptative network-based fuzzy inference system for the implementation of the two following fuzzy rules: If $x$ is $A_{1}$ AND y is $B_{1}$ THEN $f_{1}=p_{1} x+q_{1} y+r_{1}$ and If $x$ is $A_{2}$ AND y is $B_{2}$ THEN $f_{2}=p_{2} x+q_{2} y+r_{2}$.

To train the ANFIS, a data set that contains the desired input/output data of the system to be modeled is used. The modeling approach is similar to many system identification techniques: first, you hypothesize a parameterized model structure and next, thanks to an iterative and hybrid optimization method, basically a combination of least squares estimation and backpropagation gradient descent method [35], the membership function parameters are adjusted, the consequent parameters are identified and a rule base is designed, according to a chosen error criterion. The training process stops whenever the maximum iteration number is reached or the training error goal is achieved. In general, this type of modeling works well if the training data presented to the ANFIS is fully representative of the features of the data the trained system is intended to model. Checking and testing data sets allow checking the generalization capability of the resulting fuzzy inference system and avoiding overfitting [51].

\subsection{Approaches proposed for estimating the thermal diffusivity of building materials}

As previously-mentioned, three new and effective approaches are proposed for estimating the thermal diffusivity of building materials: the neuro-inverse (in this case, artificial neural networks are used for rebuilding impulse responses of building materials, their thermal diffusivity being thereafter determined by means of inverse methods), the neuronal and the neuro-fuzzy approaches.

\subsubsection{Neuro-inverse approach}

An Elman recurrent network has been trained using the glass wool, concrete, glass and stainless steel responses to a PRBS as network inputs and their respective impulse responses as targets, i.e. as desired network outputs. Let us note, and this is a key-point when rebuilding impulse responses using neural networks (or when directly estimating thermal diffusivities), that 
responses to a PRBS and impulse responses were presented to the network, during both training and validation phases, as "sequences". Whatever the response, it is considered as an entity in which all elements are connected to, and dependent upon, each other. The network's number of hidden neurons, the number of iterations carried out during its training phase and the learning rate were empirically optimized. Then, the trained network has been used for rebuilding the impulse responses of brick, plaster and granite using their responses to a PRBS as new and unknown network inputs. This is the validation phase. After rebuilding the impulse response of a material, the inversion algorithm is used for estimating its thermal diffusivity. First, the Gauss-Newton's method has been tried but the inverse problem is very ill-conditioned so we used the BoxKanemasu's method. A self-made condition has been added, close to the Box-Kanemasu's modified method, to be sure that the criterion to be minimized decreases during calculations (Figure 10).

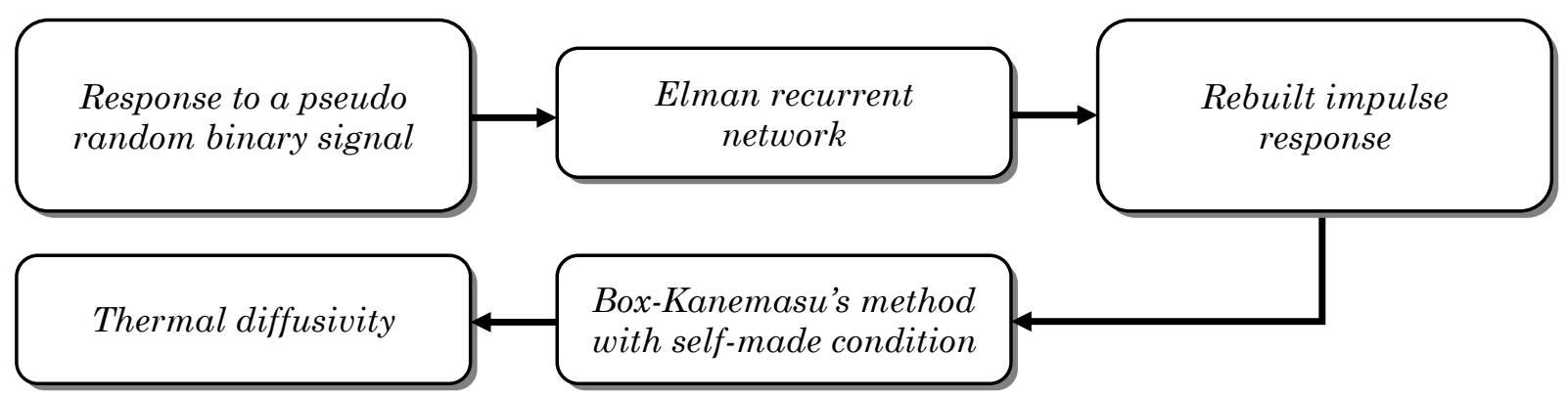

Figure 10.Neuro-inverse approach for estimating the thermal diffusivity of a building material.

\subsubsection{Neuronal approach}

Considering the results provided by the neuro-inverse approach when estimating the thermal diffusivity of building materials, a second approach, named "neuronal approach", has been tested. Using this approach, the thermal diffusivity of the considered materials is directly estimated, thanks to a multi-layer Perceptron and their respective responses to a PRBS. So, the network has been trained using the glass wool, concrete, glass and stainless steel responses to a PRBS as network inputs and their respective thermal diffusivities as targets, i.e. as desired network outputs (Figure 11). Let us note that the neuronal approach is really innovative with respect to those usually used for characterizing materials. Indeed, taking advantage of the response to a PRBS instead of its impulse response for estimating its thermal diffusivity is not a common way to do it. However, not having to rebuild the impulse response of a (fragile) material is a good thing because it allows avoiding the use of inverse methods, in some cases not very efficient (for example, when the sensitivity coefficients are weak or because ill-conditioned matrix, close to singular, cannot be well-inversed). The network's number of hidden neurons, the number of iterations carried out during its training phase and the learning rate were again empirically optimized. Then, the trained multi-layer Perceptron has been used for estimating the brick, plaster and granite's thermal diffusivities using their respective responses to a PRBS as new and unknown network inputs.

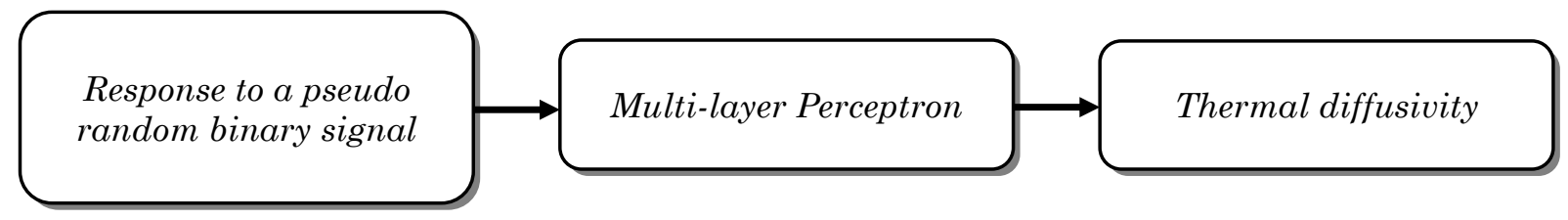

Figure 11.Neuronal approach for estimating the thermal diffusivity of a building material.

\subsubsection{Neuro-fuzzy approach}

As for both neuro-inverse and neuronal approaches, glass wool, concrete, glass and stainless steel were used to train the ANFIS, while brick, plaster and granite allowed carrying out its validation phase. As a result of the training phase, the membership function parameters are 
adjusted, the consequent parameters are identified and fuzzy rules are designed. The number of training iterations has been empirically optimized. Considering that the response to a PRBS is correlated with both the thermal diffusivity of the excited material and some of the main characteristics of the PRBS used as excitation, and taking into account expert knowledge about characterization of materials, various set of potential model inputs were tested. As a first approach, the PRBS pulses' widths and, for each of the pulses, the mean value of the obtained response, were considered. The results being disappointing, the first and the last point of the obtained response were considered instead of its mean value, but no significant improvement has been noted. Finally, the PRBS pulses' widths $\left(\Delta T=\left[\Delta T_{1} \Delta T_{2} \ldots \Delta T_{n}\right]\right)$ and the absolute value of the slopes, calculated, for each of the pulses, considering the line between the first and the median point of the obtained response $\left(s=\left[s_{1} s_{2} \ldots s_{n}\right]\right)$ were chosen and proved to be a better option (Figure 12). The thermal diffusivity of the studied material has been considered as model output (Figure 13). Let us note that, as when using the neuronal approach, the neuro-fuzzy approach allows not having to rebuild the impulse response of the considered materials. This is, again, a really innovative approach with respect to those usually used for characterizing materials.

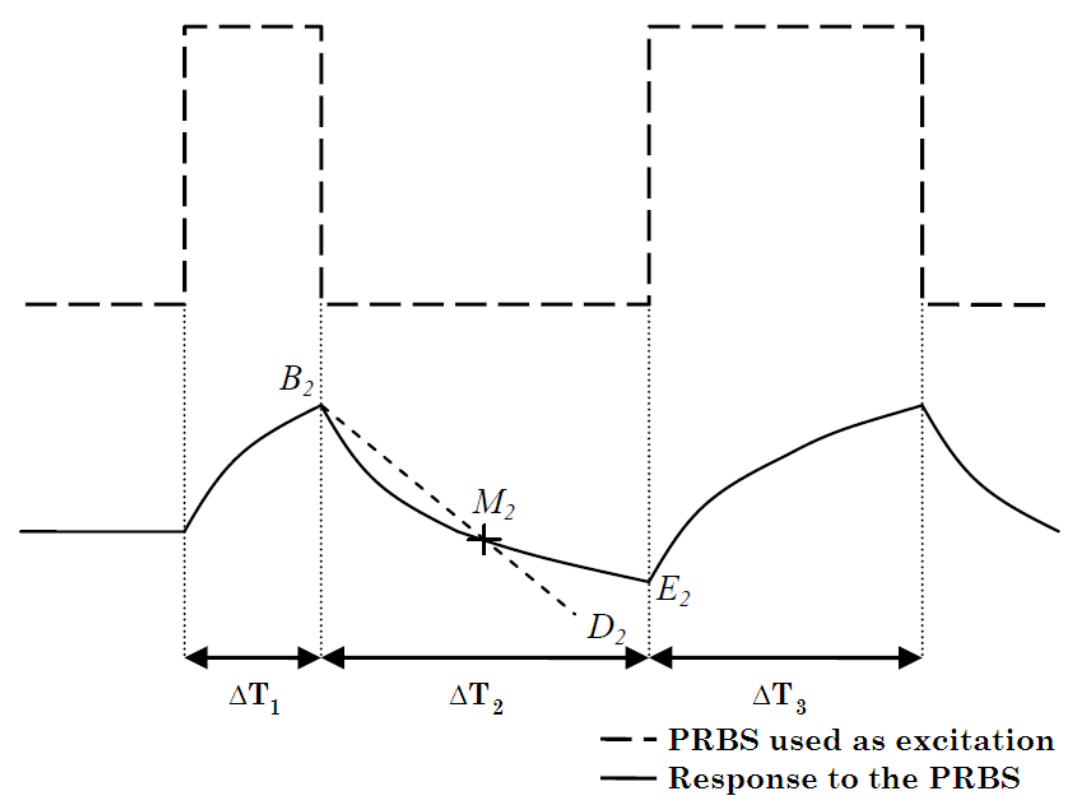

Figure 12. ANFIS input parameters. $B_{2}, M_{2}$ and $E_{2}$ are the first, median and last point of the response to the PRBS used as excitation, considering the pulse $\Delta T_{2}$.

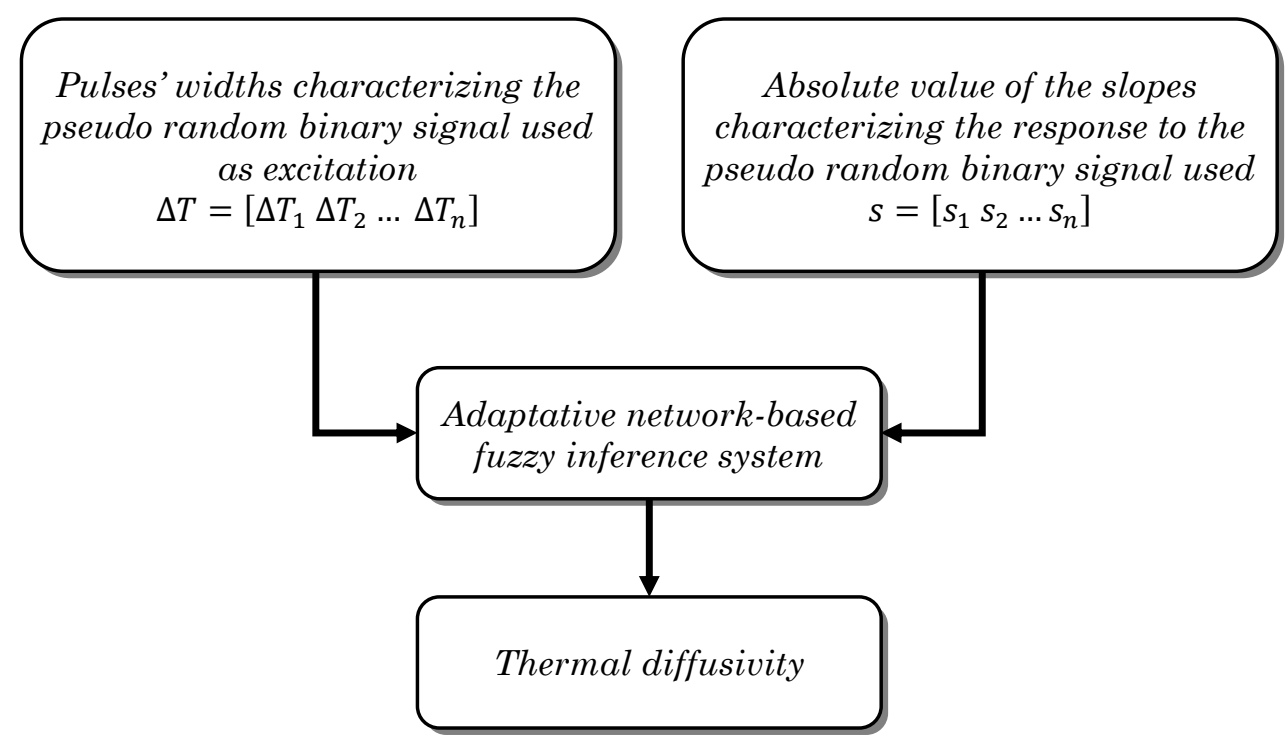

Figure 13.Neuro-fuzzy approach for estimating the thermal diffusivity of a building material. 


\section{Results}

This section of the paper deals with the results obtained using the three proposed approaches for both estimating the thermal diffusivity of building materials and contributing to the energy performance diagnosis of buildings. Rebuilt impulse responses will be compared with theoretical impulse responses using a widely-used similarity criterion (Eq.20). Mean relatives errors will also be computed when rebuilding impulse responses and estimating thermal diffusivities. Let us also note that the topology of the neural networks used and the way the training parameters have been optimized will be mentioned.

$$
F I T=100 \times\left(1-\frac{\left\|R_{\text {reb_imp }}-R_{\text {real_imp }}\right\|_{2}}{\left\|R_{\text {real_imp }}-<R_{\text {real_imp }}>\right\|_{2}}\right)
$$

\subsection{Neuro-inverse approach results}

Figures 14 to 16 present the rebuilt impulse responses of brick, plaster and granite, using a log-log scale. Let us remember that glass wool, concrete, glass and stainless steel were used to train the Elman recurrent network used. The network's hidden layer was composed of 8 neurons and 35 iterations have been carried out during the training phase. The learning rate was set to 0.3. The Levenberg-Marquardt algorithm (section 3.3.3) allowed optimizing the network's weights. Table 2 specifies, for the three validation materials, the curve fitting (FIT) and the Mean Relative Error (MRE) observed when rebuilding their respective impulse responses. Table 2 also presents, using the rebuilt impulses responses and the Box-Kanemasu's method (section 3.2.4), the result of the thermal diffusivity estimation. A Relative Error (RE) is calculated.

The results provided by the neuro-inverse approach allow, first, validating both the proposed approach and the use of artificial neural networks for rebuilding impulse responses. Whatever the validation material, the MRE is very low, ranging between $0.1 \%$ and $0.7 \%$, while the FIT is very high, ranging between $96.5 \%$ and $99.1 \%$. Let us note that taking into account the PRBS used as excitation (i.e. using it as network's second input sequence), jointly to the material response, has been considered for rebuilding impulse responses. Finally, and because one can suppose that the response to a PRBS is dependent on both the thermophysical properties of the excited material and the characteristics of the signal used as excitation, only the response to a PRBS was considered as network input sequence.

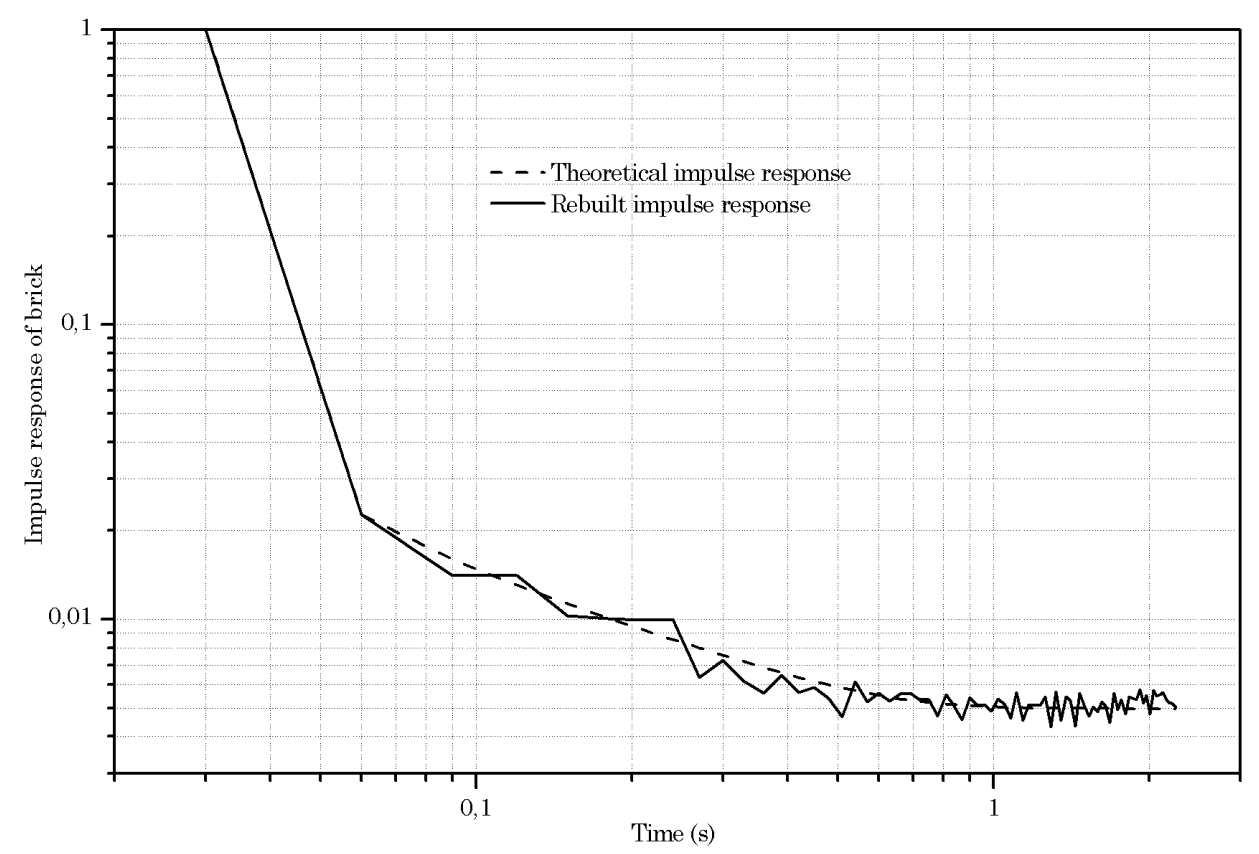

Figure 14. Rebuilding of the impulse response of brick using a previously-trained Elman recurrent network (log-log scale). 


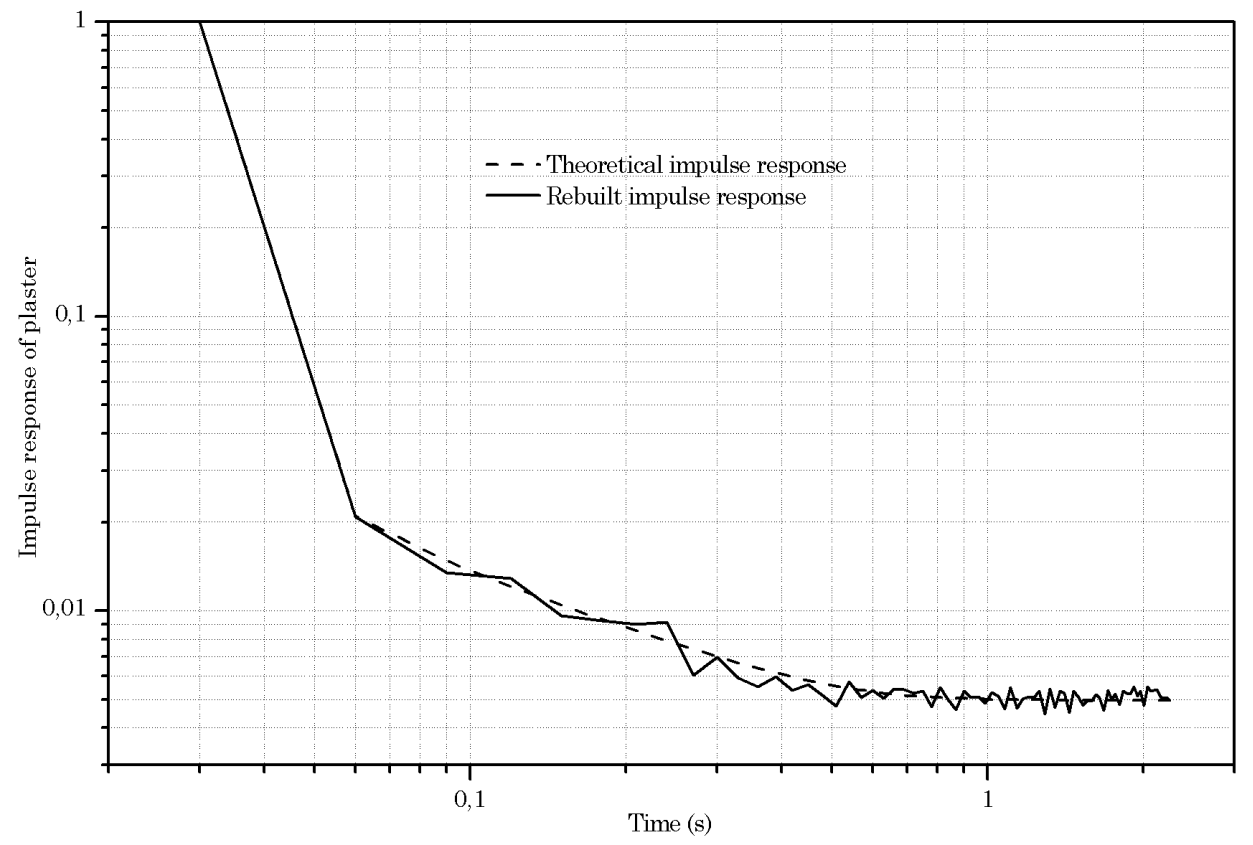

Figure 15. Rebuilding of the impulse response of plaster using a previously-trained Elman recurrent network (log-log scale).

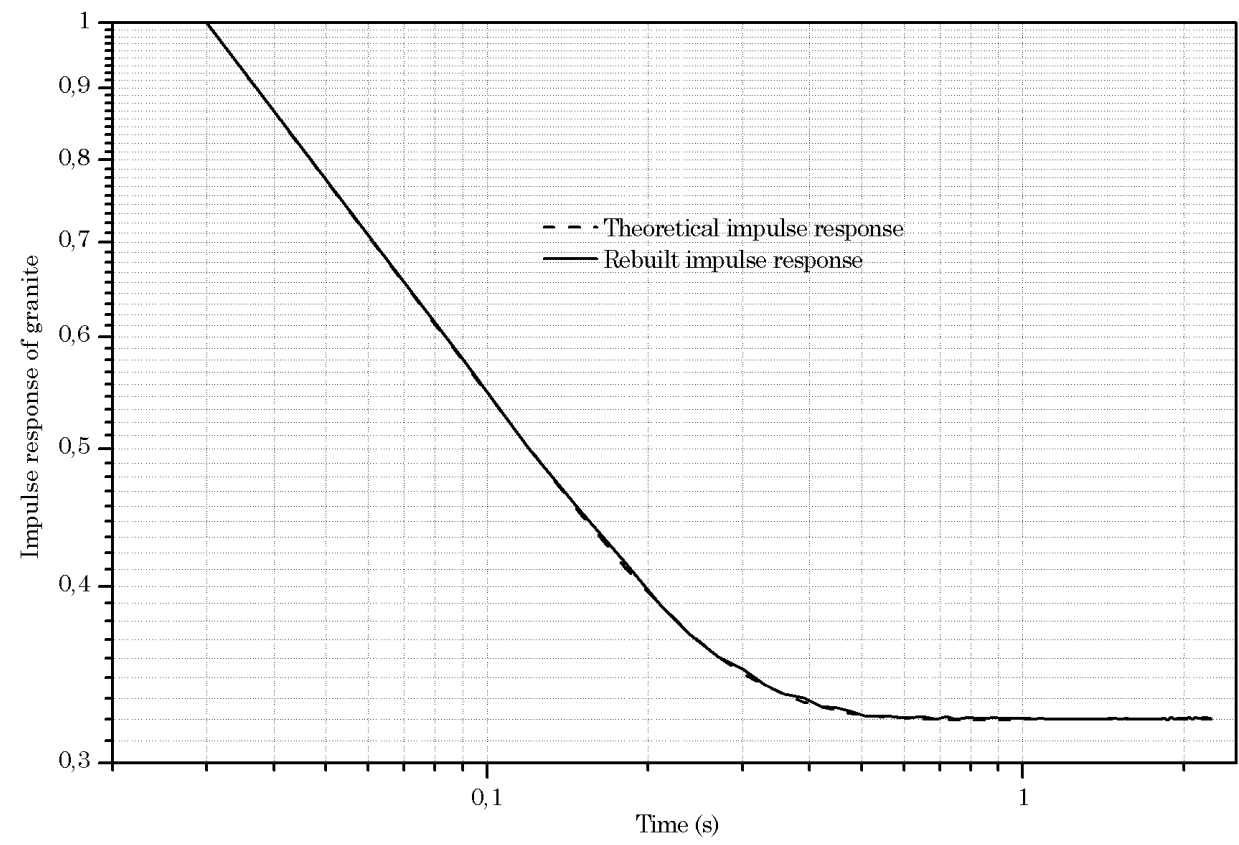

Figure 16. Rebuilding of the impulse response of granite using a previously-trained Elman recurrent network (log-log scale).

Table 2. Neuro-inverse approach results.

\begin{tabular}{|c|c|c|c|c|c|}
\hline \multirow{2}{*}{ Material } & \multicolumn{2}{|c|}{$\begin{array}{l}\text { Impulse response rebuilt } \\
\text { using an Elman network }\end{array}$} & \multicolumn{3}{|c|}{$\begin{array}{l}\text { Thermal diffusivity estimated using the Box- } \\
\text { Kanemasu's method }\end{array}$} \\
\hline & $M R E[\%]$ & FIT [\%] & $\begin{array}{l}\text { Theoretical } \\
\text { value }\left(\mathrm{m}^{2} / \mathrm{s}\right)\end{array}$ & $\begin{array}{c}\text { Estimated } \\
\text { value }\left(\mathrm{m}^{2} / \mathrm{s}\right)\end{array}$ & $R E[\%]$ \\
\hline Brick & $0.7 \%$ & $96.5 \%$ & $5.17 \times 10^{-7}$ & $4.63 \times 10^{-7}$ & $10.4 \%$ \\
\hline Plaster & $0.5 \%$ & $96.6 \%$ & $6.00 \times 10^{-7}$ & $5.06 \times 10^{-7}$ & $15.7 \%$ \\
\hline Granite & $0.1 \%$ & $99.1 \%$ & $1.10 \times 10^{-6}$ & $1.11 \times 10^{-6}$ & $<1 \%$ \\
\hline
\end{tabular}


Taking a look at Table 2, one can also observe that the Box-Kanemasu's method allows taking advantage of the rebuilt impulse responses for correctly estimating the thermal diffusivity of the considered building materials. Relative errors are ranging between less than $1 \%$ and $15.7 \%$; this leads to a MRE of about $9 \%$. One could be surprised when analyzing these late results but because both theoretical and rebuilt impulse responses contain very few points in the high sensitivity area, inverse methods are not very efficient. As a consequence, one can expect improving accuracy using both neuronal and neuro-fuzzy approaches.

\subsection{Neuronal approach results}

Let us remember that glass wool, concrete, glass and stainless steel were used to train the multi-layer Perceptron used for directly estimating the thermal diffusivity of building materials. The network's hidden layer was composed of 10 neurons and 30 iterations have been carried out during the training phase. The learning rate was set to 0.3 . The Levenberg-Marquardt algorithm (section 3.3.3) allowed optimizing the network's weights. Table 3 specifies, for the three validation materials, the result of the thermal diffusivity estimation. A Relative Error (RE) is calculated. Taking a look at Table 3, one can observe that the neuronal approach allows improving significantly the estimations' accuracy. One can note, considering the neuro-inverse results as reference results, that the neuronal approach allows reducing the RE by $40.4 \%$ (from $10.4 \%$ to $6.2 \%$ ) and $57.3 \%$ (from $15.7 \%$ to $6.7 \%$ ) when estimating the thermal diffusivities of brick and plaster respectively. Concerning granite, the RE increases from less than $1 \%$ to $4.5 \%$. One can conclude, and this is a very interesting result, that artificial neural networks are able to provide very good estimations of the thermal diffusivity of building materials, without rebuilding impulse responses, even if the sensitivity of the considered responses to a PRBS is weak. As a consequence, one can highlight that using inverse methods for estimating thermophysical properties of materials is not the only way to do it: artificial neural networks can also do it! Concerning granite, a much more diffusive material than brick and plaster, the sensitivity coefficients are weak in the used identification area, but high enough to obtain a very good estimation of its thermal diffusivity even from its rebuilt impulse response, using the Box-Kanemasu's method.

Table 3. Neuronal approach results.

\begin{tabular}{cccc}
\hline \multirow{2}{*}{ Material } & \multicolumn{3}{c}{ Thermal diffusivity estimated using a MLP } \\
\cline { 2 - 4 } & $\begin{array}{c}\text { Theoretical value } \\
\left(\mathrm{m}^{2} / \mathrm{s}\right)\end{array}$ & $\begin{array}{c}\text { Estimated value } \\
\left(\mathrm{m}^{2} / \mathrm{s}\right)\end{array}$ & $R E[\%]$ \\
\hline Brick & $5.17 \times 10^{-7}$ & $4.85 \times 10^{-7}$ & $6.2 \%$ \\
\hline Plaster & $6.00 \times 10^{-7}$ & $5.60 \times 10^{-7}$ & $6.7 \%$ \\
\hline Granite & $1.10 \times 10^{-6}$ & $1.15 \times 10^{-6}$ & $4.5 \%$ \\
\hline
\end{tabular}

\subsection{Neuro-fuzzy approach results}

As we did for developing both neuro-inverse and neuronal approaches, glass wool, concrete, glass and stainless steel were used to train the ANFIS allowing directly estimating the thermal diffusivity of building materials. The two model inputs ( $\Delta T$ and $s$ ) and their respective universes of discourse have to be characterized by means of fuzzy sets and membership functions with the aim of designing an appropriate base of fuzzy rules that best maps inputs to single output. Due to the nature of both the signal used as excitations and the obtained responses, the pulses' widths $(\Delta T)$ may be in the range $[1 \mathrm{~s} ; 8 \mathrm{~s}]$ while the absolute value of the slopes $(s)$, calculated, for each of the pulses, considering the line between the first and the median point of the obtained response, may be in the range $[0 ; 0.4675]$. Both universes of discourse have been split using only two fuzzy sets and trapezoidal membership functions. The thermal diffusivity may be in the range $\left[3 \times 10^{-7}\right.$; $\left.4 \times 10^{-6}\right]$. During the ANFIS training process, the rule extraction method allows generating rules, adjusting the shape of the input membership functions, defined, at the end of the process, as highlighted by Table 4 (considering four parameters: the left and right base points and the left and right top points), and finally identifying the coefficients of the linear output membership functions (each generated rule has one output membership function) (Table 5). 25 iterations have been carried out. Table 6 presents the obtained fuzzy rules. 
Table 4. Input membership functions' parameters.

\begin{tabular}{cccccc}
\hline $\begin{array}{c}\text { ANFIS } \\
\text { input }\end{array}$ & $\begin{array}{c}\text { Trapezoidal } \\
\text { membership function }\end{array}$ & $\begin{array}{c}\text { Left base } \\
\text { point }\end{array}$ & $\begin{array}{c}\text { Left top } \\
\text { point }\end{array}$ & $\begin{array}{c}\text { Right top } \\
\text { point }\end{array}$ & $\begin{array}{c}\text { Right base } \\
\text { point }\end{array}$ \\
\hline \multirow{2}{*}{$\Delta T$} & 1 & 1 & 1 & 3.099 & 5.9 \\
\hline \multirow{2}{*}{$s$} & 2 & 3.099 & 5.899 & 8 & 8 \\
\cline { 2 - 6 } & 1 & 0 & 0 & 0 & 0.3926 \\
\hline
\end{tabular}

Table 5. Output membership functions' parameters.

\begin{tabular}{ccccc}
\hline \multirow{2}{*}{$\begin{array}{c}\text { ANFIS } \\
\text { output }\end{array}$} & $\begin{array}{c}\text { Linear membership } \\
\text { function }\end{array}$ & $\begin{array}{c}1^{\text {st }} \\
\text { coefficient }\end{array}$ & $\begin{array}{c}2^{\text {nd }} \\
\text { coefficient }\end{array}$ & $\begin{array}{c}3^{\text {rd }} \\
\text { coefficient }\end{array}$ \\
\hline \multirow{2}{*}{$\begin{array}{c}\text { Thermal } \\
\text { diffusivity } \\
\text { (a) }\end{array}$} & $1\left(\right.$ rule $\left.^{\circ} 1\right)$ & $-3.117 \times 10^{-7}$ & $-3.855 \times 10^{-5}$ & $5.375 \times 10^{-6}$ \\
\cline { 2 - 5 } & $2\left(\right.$ rule $\left.^{\circ} 2\right)$ & $1.944 \times 10^{-7}$ & $-1.213 \times 10^{-5}$ & $5.504 \times 10^{-6}$ \\
\cline { 2 - 5 } & $3\left(\right.$ rule $\left.^{\circ} 3\right)$ & $-1.078 \times 10^{-8}$ & $-4.095 \times 10^{-5}$ & $4.345 \times 10^{-6}$ \\
\hline
\end{tabular}

Table 6. Fuzzy rules.

\begin{tabular}{ccccc}
\hline Rule & $N^{\circ} 1$ & $N^{\circ} 2$ & $N^{\circ} 3$ & $N^{\circ} 4$ \\
\hline$\Delta T$ & 1 & 1 & 2 & 2 \\
\hline$s$ & 1 & 2 & 1 & 2 \\
\hline$a$ & 1 & 2 & 3 & 4 \\
\hline
\end{tabular}

Table 7 depicts the performance of the trained ANFIS. As we did when estimating the thermal diffusivity of the chosen validation materials using the neuro-inverse and the neuronal approaches, a relative error is calculated. One can observe that the neuro-fuzzy approach allows improving significantly the estimations' accuracy. Indeed this approach provides the best results over the other proposed approaches. Taking a look at the results provided by the neuro-inverse approach (Table 2), one can highlight that the neuro-fuzzy approach allows reducing the RE by $72.1 \%$ (from $10.4 \%$ to $2.9 \%$ ) and $71.3 \%$ (from $15.7 \%$ to $4.5 \%$ ) when estimating the thermal diffusivities of brick and plaster respectively. Concerning granite, both estimations are very similar $\left(1.11 \times 10^{-6} \mathrm{~m}^{2} / \mathrm{s}\right.$ and $1.09 \times 10^{-6} \mathrm{~m}^{2} / \mathrm{s}$ when using the neuro-inverse and the neuro-fuzzy approaches respectively). Taking a look at the results provided by the neuronal approach (Table 3), one can remark that the neuro-fuzzy approach allows reducing the RE by $53.2 \%$ (from $6.2 \%$ to $2.9 \%$ ), $32.8 \%$ (from $6.7 \%$ to $4.5 \%$ ) and $75.6 \%$ (from $4.5 \%$ to $1.1 \%$ ) when estimating the thermal diffusivities of brick, plaster and granite respectively. Table 8 summarizes all the results obtained, for the three proposed approaches.

Table 7. Neuro-fuzzy approach results.

\begin{tabular}{cccc}
\hline \multirow{2}{*}{ Material } & \multicolumn{3}{c}{ Thermal diffusivity estimated using an ANFIS } \\
\cline { 2 - 4 } & $\begin{array}{c}\text { Theoretical value } \\
\left(\mathrm{m}^{2} / \mathrm{s}\right)\end{array}$ & $\begin{array}{c}\text { Estimated value } \\
\left(\mathrm{m}^{2} / \mathrm{s}\right)\end{array}$ & RE [\%] \\
\hline Brick & $5.17 \times 10^{-7}$ & $5.32 \times 10^{-7}$ & $2.9 \%$ \\
\hline Plaster & $6.00 \times 10^{-7}$ & $5.73 \times 10^{-7}$ & $4.5 \%$ \\
\hline Granite & $1.10 \times 10^{-6}$ & $1.09 \times 10^{-6}$ & $1.1 \%$ \\
\hline
\end{tabular}

Table 8. Synthesis.

\begin{tabular}{cccc}
\hline Material & $\begin{array}{c}\text { Neuro-inverse } \\
\text { approach }(\text { RE [\%]) }\end{array}$ & $\begin{array}{c}\text { Neuronal approach } \\
(\text { RE [\%]) }\end{array}$ & $\begin{array}{c}\text { Neuro-fuzzy } \\
\text { approach (RE [\%]) }\end{array}$ \\
\hline Brick & $10.4 \%$ & $6.2 \%$ & $2.9 \%$ \\
\hline Plaster & $15.7 \%$ & $6.7 \%$ & $4.5 \%$ \\
\hline Granite & $<1 \%$ & $4.5 \%$ & $1.1 \%$ \\
\hline
\end{tabular}


The obtained results validate the neuro-fuzzy approach and highlight the significant contribution of expert knowledge, which has been considered using, as ANFIS inputs, characteristics of both the PRBS used as excitation and the response to this excitation. Again, these results confirm, first, that artificial intelligence tools are useful for characterizing materials and, secondly, that rebuilding impulse responses for estimating thermophysical properties is not mandatory: one can directly estimate the thermal diffusivity of materials using artificial neural networks or neurofuzzy systems and responses to pseudo random binary signals. As a consequence, and this is the main result of the study, one can maintain that both neuronal and neuro-fuzzy approaches allow not using inverse methods (these methods are not very efficient when impulse responses contain very few points in the high sensitivity area) and correlation analysis techniques (as previouslymentioned, these techniques are very complex to apply, require a large computational time and are, in some cases, not very efficient).

\section{Conclusion and perspectives}

With the building sector being one of the largest sectors of energy consumption in Europe, and consequently one of the major causes of greenhouse gas emissions and therefore of global warming, reliable and robust tools are needed for carrying out an advanced energy performance diagnostic and issuing energy performance certificates for buildings. Because invisible defaults, like, for example, non emerging cracks or delaminations, completely spoil the insulating qualities of buildings, owners (and future owners) would be pleased to locate these defaults. Whatever the situation, the challenge is the same: being able to locate invisible things under a layer of plaster or similar material, which amounts to locate inhomogeneities in a homogeneous medium. These defaults locally modifying the global thermophysical properties of the medium, they can be located by an in-situ estimation of these properties.

The present work deals with new and effective approaches, alternative to commonly-used (and not always very efficient) approaches and based on artificial intelligence tools, for estimating the thermal diffusivity of building materials, using thermograms obtained with a non-destructive photothermal method. Three approaches are proposed, based on responses to a PRBS: rebuilding impulses responses using an Elman recurrent network and then estimating thermal diffusivities thanks to an inverse method (neuro-inverse approach) and directly estimating thermal diffusivities using a multi-layer Perceptron (neuronal approach) or an adaptative network-based fuzzy inference system (neuro-fuzzy approach). The first conclusion of the work is that artificial intelligence tools are useful for characterizing materials. Indeed, whatever the proposed approach, the results are very satisfactory. Let us note that the best results are provided by the neuro-fuzzy approach, highlighting both the significant contribution of expert knowledge and the fact that rebuilding impulse responses for estimating thermophysical properties is not mandatory. One can directly estimate the thermal diffusivity of building materials from responses to PRBS, using artificial intelligence tools. Future works will now focus, first, on considering more materials, and as a consequence more thermal behaviors, for developing global models about thermal diffusivity estimation. In particular, we will be interested in organic building materials, such as, for example, wood or clay. Among all the materials used for developing and testing the proposed approaches, only glass wool is such a material. Characterizing dual-layer materials will also be considered. Finally, future works will also centre on, first, validating experimentally the developed models and, secondly, on extending the range of application of these models to other interesting thermophysical properties (such as thermal effusivity or thermal contact resistance).

\section{References}

[1] French Environment and Energy Management Agency, Les chiffres clés du bâtiment en 2006 (Building Sector Key Figures in 2006), ADEME Publications, 2007.

[2] Directive 2002/91/EC of the European Parliament and the Council of the 16 December 2002 on the energy performance of buildings, Official Journal of the European Communities, 2002.

[3] Y.-K. Juan, P. Gao, J. Wang, A hybrid decision support system for sustainable office building and energy performance improvement, Energy and Buildings 42 (3) (2010) 290-297.

[4] A.G. Entrop, H.J.H. Brouwers, A.H.M.E. Reinders, Evaluation of energy performance indicators and financial aspects of energy saving techniques in residential real estate, Energy and Buildings 42 (5) (2010) 618-629. 
[5] Market Observatory for Energy, Europe's energy position. Present \& future, 2008.

[6] Décret n²006-592 du 24 mai 2006 relatif aux caractéristiques thermiques et à la performance énergétique des constructions, Official Journal of French Republic, 2006.

[7] Décret $n^{\circ} 2006-1147$ du 14 septembre 2006 relatif au diagnostic de performance énergétique et à l'état de l'installation intérieure, Official Journal of French Republic, 2006.

[8] M. Ordenes, R. Lamberts, S. Güths, Estimation of thermophysical properties using natural signal analysis with heat and moisture transfer model, Energy and Buildings 41 (12) (2009) 1360 1367.

[9] B. Yesilata, P. Turgut, A simple dynamic measurement technique for comparing thermal insulation performances of anisotropic building materials, Energy and Buildings 39 (9) (2007) 1027-1034.

[10] S. Brahim, Radiométrie photothermique sous excitation aléatoire : application à la mesure de propriétés thermophysiques, Ph.D. thesis, University of Reims Champagne-Ardenne, France, 2008.

[11] J.L. Bodnar, S. Brahim, A. Boutemy, J.C. Cantone, P. Grossel, Contrôle non destructif par radiométrie photothermique sous excitation aléatoire : principe et exemples d'application, Congrès Annuel de la Société Française de Thermique, Reims, France, 2005.

[12] W.J. Parker, W.J. Jenkins, C.P. Butler, G.L. Abbott, Flash method for determining thermal diffusivity, heat capacity and thermal conductivity, Journal of Applied Physics 32 (9) (1961) 16791684.

[13] J. Max, J.L. Lacoume, Méthodes et techniques de traitement du signal, Editions Dunod, Paris, France, 2000.

[14] A. Yezioro, B. Dong, F. Leite, An applied artificial intelligence approach towards assessing building performance simulation tools, Energy and Buildings 40 (4) (2008) 612-620.

[15] B. Paris, J. Eynard, S. Grieu, T. Talbert, M. Polit, Heating control schemes for energy management in buildings, Energy and Buildings 42 (10) (2010) 1908-1917.

[16] H. Ohno, T. Suzuki, K. Aoki, A. Takahasi, G. Sugimoto, Neural network control for automatic braking control system, Neural Networks 7 (8) (1994) 1303-1312.

[17] K.P. Venugopal, A.S. Pandya, R. Sudhakar, A recurrent neural network controller and learning algorithm for the on-line learning control of autonomous underwater vehicles, Neural Networks 7 (5) (1994) 833-846.

[18] I. Flood, P. Christopholis, Modelling construction processes using artificial neural networks, Automation in Construction 4 (4) (1996) 307-320.

[19] S. Grieu, A. Traoré, M. Polit, J. Colprim, Prediction of parameters characterizing the state of a pollution removal biologic process, Engineering Applications of Artificial Intelligence 18 (5) (2005) 559-573.

[20] S. Grieu, S., F. Thiéry, A. Traoré, T.P. Nguyen, M. Barreau, M. Polit, KSOM and MLP neural networks for on-line estimating the efficiency of an activated sludge process, Chemical Engineering Journal 116 (1) (2006) 1-11.

[21] W.J. Zhang, C.J. Bai, G.D. Liu, Neural network modelling of ecosystems: A case study on cabbage growth system, Ecological Modelling 201 (3-4) (2007) 317-325.

[22] Y. Zuo, Y. Wang, X. Liu, S.X. Yang, L. Huang, X. Wu, Z. Wang, Neural network robust Ho tracking control strategy for robot manipulators, Applied Mathematical Modelling 34 (7) (2010) $1823-1838$

[23] G. Dreyfus, J.M. Martinez, M. Samuelides, M.B. Gordon, F. Badran, S. Thiria, Apprentissage statistique. Réseaux de neurones. Cartes topologiques. Machines à vecteurs supports, Editions Eyrolles, Paris, France, 2008.

[24] J.S.R. Jang, ANFIS: Adaptative-Network-Based Fuzzy Inference System, IEEE Transactions on Systems, Man, and Cybernetics 23 (3) (1993) 665-685.

[25] K.D. Maglic, A. Cezairliyan, V.E. Peletsky, Compendium of thermophysical property measurement methods 1: survey of measurement techniques, Plenum Publishers, New York, USA, 1984.

[26] K.D. Maglic, A. Cezairliyan, V.E. Peletsky, Compendium of thermophysical property measurement methods 2: recommended measurement techniques and practices, Plenum Publishers, New York, USA, 1992. 
[27] C. Gervaise, Caractérisation thermique multi-échelles de revêtements réfractaires. Comportement sous excitation périodique et identification par méthode inverse, Ph.D. thesis, University Paul Sabatier, Toulouse, France, 1999.

[28] J.L. Bodnar, S. Brahim, Contrôle non destructif par radiométrie photothermique aléatoire, Thermogram'03 conférence, Sénart, France, 2003.

[29] J. Auvray, Identification de processus, Techniques de l'ingénieur, Fiche R306, 1994.

[30] O. Faugeroux, Caractérisation thermophysique de revêtement de protection thermomécanique par méthode photothermique impulsionnelle, Ph.D. thesis, University of Perpignan, France, 2001.

[31] J.V. Beck, K.J. Arnold, Parameters estimation in Engineering and Sciences, John Wiley \& Sons Ltd, New York, USA, 1977.

[32] R. Rosenblatt, The perceptron: a perceiving and recognizing automaton, Project PARA, Report 85-460-1, Cornell Aeronautical Laboratory, 1957.

[33] W.S. McCullogh, W. Pitts, A logical calculus of the ideas immanent in nervous activity, Bull. Math. Biophys. 5 (1943) 115-133.

[34] K. Hornik, M. Stinchcombe, H. White, Multi-layer feedforward networks are universal approximation, Neural Networks 2 (1989) 359-366.

[35] C. Charalambous, Conjugate gradient algorithm for efficient training of artificial neural networks, IEEE Proc. 139 (1992) 301-310.

[36] H. Demuth, M. Beale, Neural Network Toolbox: For Use with Matlab, The MathWorks, Inc, Natick, MA, USA, 1992.

[37] D. Mandic, J. Chambers, Recurrent neural networks for prediction: learning algorithms, architectures and stability, Adaptative and Learning Systems for Signal Processing, Communications and Control Series, John Wiley \& Sons Ltd, New York, USA, 2001.

[38] J.L. Elman, Finding structures in time, Cognitive Sciences 14 (1990) 179-211.

[39] M.T. Hagan, M. Menhaj, Training feedforward networks with the Marquardt algorithm, IEEE Trans. Neural Networks 5 (1994) 989-993.

[40] C.T. Lin, C.S.G. Lee, Neural Fuzzy Systems: A Neuro-Fuzzy Synergism to Intelligent Systems, Upper Saddle River, NJ: Prentice Hall, 1996.

[41] A. Abraham, Adaptation of Fuzzy Inference System Using Neural Learning, Fuzzy System Engineering: Theory and Practice, Nadia Nedjah et al. (Eds.), Studies in Fuzziness and Soft Computing, Springer Verlag Germany 3 (2005) 53-83.

[42] J.J. Buckley, Y. Hayashi, Fuzzy neural networks: a survey, Fuzzy Sets and Systems 66 (1994) 1-13.

[43] J.J. Buckley, Y. Hayashi, Neural networks for fuzzy systems, Fuzzy Sets and Systems 71 (1995) 265-276.

[44] L. Zadeh, Fuzzy sets, Information and Control 8 (1965) 338-353.

[45] L. Zadeh, Outline of a new approach to the analysis of complex systems and decision processes, IEEE Transactions on Systems, Man, and Cybernetics 3 (1973) 28-44.

[46] T. Takagi, M. Sugeno, Derivation of fuzzy control rules from human operator's control actions, Proc. IFAC Symp. Fuzzy Inform., Knowledge Representation and Decision Analysis, pp. $55-60,1983$

[47] C.C. Lee, Fuzzy logic in control systems: Fuzzy logic controller - Part I, IEEE Transactions on Systems, Man, and Cybernetics 20 (1990) 404-418.

[48] C.C Lee, Fuzzy logic in control systems: Fuzzy logic controller - Part II, IEEE Transactions on Systems, Man, and Cybernetics 20 (1990) 419-435.

[49] J.S.R. Jang, C.T. Sun, Neuro-fuzzy modeling and control, IEEE Proceedings 83 (3) (1995) 378406.

[50] E.P. Klement, R. Mesiar, E. Pap, Triangular norms, Kluwer Academics Publishers, Dordrecht, The Netherlands, 2000.

[51] C. Schittenkopf, G. Deco, W. Brauer, Two strategies to avoid overfitting in feedforward networks, Neural Networks 10 (1997) 505-516. 